\title{
Robust Weighted Timed Automata and Games
}

\author{
Patricia Bouyer, Nicolas Markey, Ocan Sankur \\ LSV, CNRS \& ENS Cachan, France
}

\begin{abstract}
Weighted timed automata extend timed automata with cost variables that can be used to model the evolution of various quantities. Although cost-optimal reachability is decidable (in polynomial space) on this model, it becomes undecidable on weighted timed games. This paper studies cost-optimal reachability problems on weighted timed automata and games under robust semantics. More precisely, we consider two perturbation game semantics that introduce imprecisions in the standard semantics, and bring robustness properties w.r.t. timing imprecisions to controllers. We give a polynomial-space algorithm for weighted timed automata, and prove the undecidability of cost-optimal reachability on weighted timed games, showing that the problem is robustly undecidable.
\end{abstract}

\section{Introduction}

Weighted timed automata. Weighted timed automata are an extension of timed automata [2], introduced in [4,7], which allow to express and solve optimization problems on timed systems. In this model, timed automata are enriched with cost variables whose values grow with given constant derivatives in each location. This allows one to describe systems with timing constraints while specifying the evolution of some resources, such as energy. The cost-optimal reachability problem was studied in $[4,7,8]$, and the problem was shown to be PSPACE-complete [8]. The model naturally extends to timed games. Although some partial decidability results for weighted timed games have been proposed in $[1,10]$, the problem is undecidable in the general case $[12,9]$ even for a fixed number of clocks.

Robustness. Timed automata and their extensions are abstract formalisms to describe models of real-time systems. Consequently, these formalisms make idealistic assumptions, such as the perfect continuity of the clocks, their high precision, and instantaneous reaction of the system. One side effect of such idealistic semantics is that they allow easily encoding of undecidable problems. In fact, the undecidability proofs on weighted timed games of $[12,9]$ rely on the ability of timed automata to distinguish infinitely precise clock values, and modify these values with high precision. It is believed that some of the undecidability results in the literature can be overcome by introducing fuzziness in the semantics, making it impossible to encode complex (undecidable) languages, see for instance $[3,5,17]$.

In this paper, we investigate how adding imprecisions to the semantics affects the (un)decidability of the cost-optimal reachability problem in weighted timed

This work was partly supported by ANR project ImpRo (ANR-10-BLAN-0317), by ERC Starting grant EQualIS (308087) and by European project Cassting (FP7-ICT-601148). 
automata and games. We consider two prominent perturbation semantics from [14, $11,22]$, where perturbations are modeled as a game between a controller which chooses delays and edges, and an environment which perturbs the chosen delay by a (parametrized) bounded amount. The problem consists in deciding whether the controller has a winning strategy ensuring a given objective for a sufficiently small value of the parameter. Such a winning strategy is then robust, since it can ensure winning even if the moves it suggests are perturbed by a bounded amount. Two variants have been considered: in the excess-perturbation semantics, the controller is only required to suggest delays and edges whose guards are satisfied after the delay, while the guard can be violated after perturbations. This semantics allows one to design systems with simple timing constraints (for instance, using equalities), and synthesize robust strategies afterwards, taking into account new behaviors due to imprecisions. The conservative perturbation semantics requires the guards to be satisfied after any perturbation. This semantics appears naturally for instance in applications where lower and upper bounds on task execution times are given and need to be respected strictly.

For timed automata, robust reachability is EXPTIME-complete for the excessperturbation semantics [11], while robust reachability and safety are PSPACEcomplete for the conservative perturbation semantics [22]. Here, we apply both semantics to weighted timed automata and games and study the problem of deciding an upper bound on the limit-cost of a winning strategy for reachability objectives for the controller. The limit-cost refers to the limit of the cost achieved by a given strategy, when the bound $\delta$ on the perturbations goes to zero. In fact, the cost of a given path can slightly increase (or decrease) due to perturbations on the delays, but only by an amount proportional to $\delta$. Thus, the limit-cost allows us to concentrate on the bound that could be achieved if this effect is discarded. On weighted timed automata, we prove that the problem is PSPACE-complete in the conservative perturbation semantics, by adapting the corner-point abstraction [8]. In the excess-perturbation semantics, we show, perhaps surprisingly, that the problem becomes undecidable on weighted timed automata. This goes against the idea that introducing perturbations could render problems more tractable. On weighted timed games, our results are negative: in both excess- and conservative perturbation semantics, cost optimal reachability remains undecidable on weighted timed games, even when the number of clocks is fixed and the constants are bounded. We also prove the undecidability of the problem for fixed parameter $\delta$ on weighted timed games. Hence, similarly to "robust undecidability" results of [19] on timed automata, we establish that cost-optimal reachability on weighted timed games is robustly undecidable, for the considered semantics.

Related Work. The work [18] attempted to introduce fuzziness in the semantics of timed automata, via a topological semantics, with the hope of extending the decidability results. However timed language inclusion turned out to be still undecidable [19]. Another related line of work is that of [20,16, 15], which consists in modeling imprecisions by enlarging all clock constraints of the automaton by some parameter $\delta$, that is, transforming each constraint of the form $x \in[a, b]$ into $x \in[a-\delta, b+\delta]$. One analyzes the resulting system with a worst case 
approach. The game semantics we consider allow the system to observe and react to perturbations. The dual notion of shrinking was considered in [21] in order to study whether any significant behavior is lost when guards are shrunk.

\section{Preliminaries}

Game structures. A (two-player) game structure is a tuple $\mathcal{T}=\left(S, s_{0}, M_{1}, M_{2}\right.$, $T_{1}, T_{2}, \mathrm{jt}$ ), where $S$ is a set of states, $s_{0} \in S$ is the initial state, $M_{i}$ is the set of moves of Player $i, T_{i} \subseteq S \times M_{i}$ is the enabling condition for Player $i$, and jt: $S \times M_{1} \times M_{2} \rightarrow S$ the joint transition function. We assume that each $M_{i}$ contains a distinguished element $\perp$ called the empty move, and that jt $(s, \perp, \perp)=s$ for any $s \in S$. A run of $\mathcal{T}$ is a finite or infinite sequence $q_{1} e_{1} q_{2} e_{2} \ldots$ of alternating states $q_{i} \in S$, and pairs of moves $e_{i}=\left(m_{1}, m_{2}\right) \in M_{1} \times M_{2}$, such that for all $i \geq 1$, we have $\left(q_{i}, m_{\iota}\right) \in T_{\iota}$ for $\iota \in\{1,2\}$, and $q_{i+1}=\mathrm{jt}\left(q_{i}, e_{i}\right)$. For any finite run $\rho$, let $|\rho|$ denote its length, that is, the number of states it contains. For any natural number $1 \leq i \leq|\rho|$, let $\operatorname{state}_{i}(\rho)$ the $i$-th state of $\rho$, and $\operatorname{trans}_{i}(\rho)$ its $i$-th transition. We let first $(\rho)=\operatorname{state}_{1}(\rho)$, and last $(\rho)=\operatorname{state}_{|\rho|}(\rho)$. We also denote by $\rho_{i \ldots j}$ the sub-run of $\rho$ between states of indices $i$ and $j$.

A strategy for Player $i$ is a function $f$ that maps each finite run $h$ to a move $M_{i}$, such that $(\operatorname{last}(h), f(h)) \in T_{i}$. A run $\rho$ is compatible with strategies $f$ and $g$ of Players 1 and 2, if state st+1 $(\rho)=\operatorname{jt}\left(\rho_{1 \ldots i},\left(f\left(\rho_{1 \ldots i}\right), g\left(\rho_{1 \ldots i}\right)\right)\right)$ for all $i \geq 1$. Given strategies $f$ and $g$ for Players 1 and 2 resp., the outcome of the pair $(f, g)$ in $\mathcal{T}$, denoted by Outcome $\mathcal{T}(f, g)$ is the unique infinite run that is compatible with both strategies. Let $S_{i}(\mathcal{T})$ denote the set of the strategies of Player $i$ in $\mathcal{T}$.

Weighted timed automata and games. Given a finite set of clocks $\mathcal{C}$, we call valuations the elements of $\mathbb{R}_{>0}^{\mathcal{C}}$. For a subset $R \subseteq \mathcal{C}$ and a valuation $\nu, \nu[R \leftarrow 0]$ is the valuation defined by $\nu[\bar{R} \leftarrow 0](x)=\nu(x)$ for $x \in \mathcal{C} \backslash R$ and $\nu[R \leftarrow 0](x)=0$ for $x \in R$. Given $d \in \mathbb{R}_{\geq 0}$ and a valuation $\nu$, the valuation $\nu+d$ is defined by $(\nu+d)(x)=\nu(x)+d$ for all $x \in \mathcal{C}$. We extend these operations to sets of valuations in the obvious way. We write $\mathbf{0}$ for the valuation that assigns 0 to every clock.

An atomic clock constraint is a formula of the form $k \preceq x \preceq^{\prime} l$ or $k \preceq x-y \preceq^{\prime} l$ where $x, y \in \mathcal{C}, k, l \in \mathbb{Z} \cup\{-\infty, \infty\}$ and $\preceq, \preceq^{\prime} \in\{<, \leq\}$. A guard is a conjunction of atomic clock constraints. A valuation $\nu$ satisfies a guard $g$, denoted $\nu \models g$, if all constraints are satisfied when each $x \in \mathcal{C}$ is replaced with $\nu(x)$. We write $\Phi_{\mathcal{C}}$ for the set of guards built on $\mathcal{C}$.

Definition 1. A weighted timed game $(W T G) \mathcal{A}$ is a tuple $\left(\mathcal{L}, \ell_{0}, \mathcal{C}, \mathcal{I}, E_{1}, E_{2}, \mathcal{S}\right)$, where $\mathcal{L}$ is a finite set of locations, $\mathcal{C}$ is a finite set of clocks, $\mathcal{I}: \mathcal{L} \rightarrow \Phi_{\mathcal{C}}$ assigns an invariant to every location, $E_{1}, E_{2} \subseteq \mathcal{L} \times \Phi_{\mathcal{C}} \times 2^{\mathcal{C}} \times \mathcal{L}$ are sets of edges, $\ell_{0} \in \mathcal{L}$ is the initial location, and $\mathcal{S}: \mathcal{L} \rightarrow \mathbb{Z}$ is the slope function ${ }^{1}$. For any edge $e=\left(\ell, g, R, \ell^{\prime}\right), g$ is the guard of the edge, and $R$ its reset set. An edge $e=\left(\ell, g, R, \ell^{\prime}\right)$ is also written as $\ell \stackrel{g, R}{\longrightarrow} \ell^{\prime}$. A weighted timed automaton (WTA) is a WTG of the form $\left(\mathcal{L}, \ell_{0}, \mathcal{C}, \mathcal{I}, E_{1}, \emptyset, \mathcal{S}\right)$.

\footnotetext{
${ }^{1}$ We do not introduce discrete weights on transitions, but all our results would also hold in that setting.
} 
Weighted timed games define game structures similarly to timed games [6]: the guards of the edges enable or disable the transitions, and the reset set determines the update after the transition is taken by resetting the clocks belonging to the set. Intuitively, the edges $E_{i}$ are controlled by Player $i$. Moreover, the state space contains the value of a cost variable, denoted cost, which grows with derivative $\mathcal{S}(\ell)$ at any location $\ell$.

In this paper, we assume that all clocks are bounded above by a constant, i.e., the invariant at each location imposes some upper bound on all clocks.

Formally, the exact semantics of a WTG $\mathcal{A}$ is a game structure $\llbracket \mathcal{A} \rrbracket=$ $\left(S, s_{0}, M_{1}, M_{2}, T_{1}, T_{2}, \mathrm{jt}\right)$. The state space is $S=\left\{(\ell, \nu, c) \mid \ell \in \mathcal{L}, \nu \in \mathbb{R}_{>0}^{\mathcal{C}}, c \in\right.$ $\mathbb{R}, \nu \models \mathcal{I}(\ell)\}$. The initial state is $s_{0}=\left(\ell_{0}, \mathbf{0}, 0\right)$. The moves are defined by $M_{i}=\mathbb{R} \times E_{i} \cup\{\perp\}$ whose components are pairs of a delay and a Player- $i$ edge. A pair $(d, e) \in \mathbb{R} \times E_{i}$ is said enabled at $(\ell, \nu) \in \mathcal{L} \times \mathbb{R}_{\geq 0}^{\mathcal{C}}$ whenever, writing $e=\left(\ell_{1}, g, R, \ell_{2}\right)$, we have $\ell=\ell_{1}, \nu=\mathcal{I}\left(\ell_{1}\right), \nu+d=\mathcal{I}\left(\ell_{1}\right), \nu+d=g$, and $(\nu+d)[R \leftarrow 0] \models \mathcal{I}\left(\ell_{2}\right)$. The enabling condition $T_{i}((\ell, \nu, c),(d, e))$ holds if, and only if $(d, e)$ is enabled at $(\ell, \nu)$. Note that $T_{i}((\ell, \nu, c), \perp)$ holds at any state $(\ell, \nu, c)$. The joint transition function is defined as follows. Given $d_{i} \geq 0$, and edges $e_{i}=\left(\ell, g_{i}, R_{i}, \ell_{i}\right) \in E_{i}$, we have jt $\left((\ell, \nu, c),\left(d_{1}, e_{1}\right),\left(d_{2}, e_{2}\right)\right)=\left(\ell_{i_{0}},\left(\nu+d_{i_{0}}\right)\left[R_{i_{0}} \leftarrow\right.\right.$ $\left.0], c+d_{i_{0}} \mathcal{S}(\ell)\right)$, where $i_{0}=1$ if $d_{1} \leq d_{2}$, and $i_{0}=2$ otherwise. Moreover, jt $\left((\ell, \nu, c),\left(d_{1}, e_{1}\right), \perp\right)=\left(\ell_{1},\left(\nu+d_{1}\right)\left[R_{1} \leftarrow 0\right], c+d_{1} \mathcal{S}(\ell)\right)$, and symmetrically.

Example 1. Figure 1 displays an example of a weighted timed game. Plain (resp. dashed) arrows are for Player 1 (resp. Player 2) edges. The slopes are indicated above each state. A strategy for Player 1 is to suggest a delay of 1 and choose the edge from $\ell_{1}$ to $\ell_{2}$. This prevents Player 2 from going down to location $\ell_{5}$, where the cost of accepting is $12-o(\delta)$. From location $\ell_{2}$, Player 1 can go to $\ell_{4}$, from where a target location is reached with cost 7 .

Regions, Vertices. We assume familiarity with regions (see [2]). We recall that the region automaton of a timed automaton $\mathcal{A}$ is a finite automaton, denoted $\mathcal{R}(\mathcal{A})$, with states $(\ell, r)$, where $\ell$ is a location, and $r$ a region. Let us write $\operatorname{loc}((\ell, r))=\ell$. There is a transition $(\ell, r) \stackrel{\text { delay }}{\longrightarrow}\left(\ell, r^{\prime}\right)$ iff some valuation in $r^{\prime}$ can be reached by a time delay from some valuation in $r$. We have $(\ell, r) \stackrel{e}{\rightarrow}\left(\ell^{\prime}, r^{\prime}\right)$, for an edge $e=\left(\ell, g, R, \ell^{\prime}\right)$ iff all valuations of $r$ satisfy $g$, and $r^{\prime}=r[R \leftarrow 0]$. A path of $\mathcal{R}(\mathcal{A})$ is a sequence $q_{1} t_{1} q_{2} t_{2} \ldots$ where for all $i \geq 1, q_{i}=\left(\ell_{i}, r_{i}\right)$ for some location $\ell_{i}$ and region $r_{i}$, and $t_{i}$ is either an edge or delay. We say that a run $\rho$ of $\mathcal{A}$ follows a path $\pi=q_{1} t_{1} \ldots$ of $\mathcal{R}(\mathcal{A})$ if for any $i \geq 1$, if we write $\left(\ell_{i}, r_{i}\right)=\operatorname{state}_{i}(\pi)$,

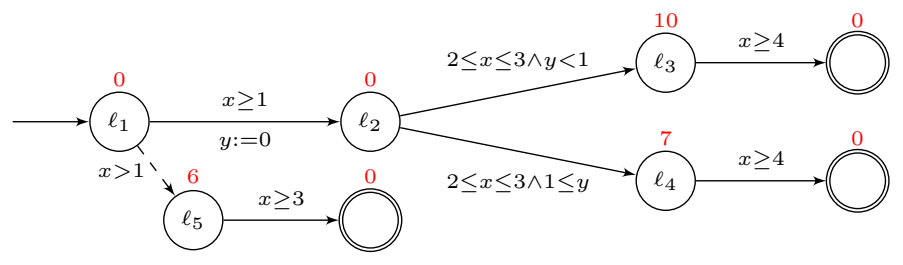

Fig. 1. Example of a WTG 
then $\operatorname{state}_{i}(\rho)=\left(\ell_{i}, \nu_{i}\right)$ for some $\nu_{i} \in r_{i}$, and moreover, $\operatorname{trans}_{i}(\pi)=$ delay implies that $\operatorname{trans}_{i}(\rho) \in \mathbb{R}_{\geq 0}$, and $\operatorname{trans}_{i}(\pi)=\operatorname{trans}_{i}(\rho)$ otherwise.

A valuation with integer coordinates is called a vertex. For any region $r$, let us denote by $\mathcal{V}(r)$ the set of vertices that belong the topological closure of $r$. A region is non-punctual if for some $\nu \in r$, and $\epsilon>0, \nu+[-\epsilon, \epsilon] \subseteq r$. It is punctual otherwise. A non-punctual path of the region automaton is a path of $\mathcal{R}(\mathcal{A})$ where all regions reached after a delay are non-punctual.

\section{Robust Cost-Optimal Reachability}

We now define two perturbed semantics for weighted timed games. These semantics were first studied in $[13,11]$ for timed automata and games in order to synthesize robust strategies. We adapt these here to WTG and formalize cost-optimal reachability problems.

Perturbed semantics. We will call Player 1 Controller, and Player 2 Perturbator. The idea behind the perturbed semantics is to give Perturbator the additional power of perturbing the delays chosen by Controller by some bounded amount $\delta$ (in that sense, the perturbed semantics becomes asymmetric). In this setting, a winning strategy for Controller is then robust to perturbations in the time delays. Informally, at any state $(\ell, \nu, c)$, both players suggest a delay and an edge. If Perturbator suggests a shorter delay, then the suggested delay and edge is taken. If Controller suggests the shorter delay $d$ and edge $e$, then the system moves to an intermediate state $(\ell, \nu, c, d, e)$, from which Perturbator chooses $\epsilon \in[-\delta, \delta]$, and the edge $e$ is taken after a delay of $d+\epsilon$; the cost then increases by $(d+\epsilon) \cdot \mathcal{S}(\ell)$. We require both players to only suggest delays no smaller than $\delta$, to model the fact that the system is not infinitely fast. We will formally define two perturbed semantics based on the above ideas; they will differ on the satisfaction or not of the guards after the delay has been perturbed by $\epsilon$.

Formally, given $\delta>0$, the $\delta$-excess perturbation semantics of a WTG $\mathcal{A}=$ $\left(\mathcal{L}, \ell_{0}, \mathcal{C}, \mathcal{I}, E_{1}, E_{2}, \mathcal{S}\right)$ is a game structure $\mathcal{G}_{\delta}^{e}(\mathcal{A})=\left(S^{\prime}, s_{0}^{\prime}, M_{1}^{\prime}, M_{2}^{\prime}, T_{1}^{\prime}, T_{2}^{\prime}, \mathrm{jt}^{\prime}\right)$, where $S^{\prime}=S \cup S \times \mathbb{R}_{\geq 0} \times E_{1}$, with $S=\left\{(\ell, \nu, c) \mid \ell \in \mathcal{L}, \nu \in \mathbb{R}_{\geq 0}^{\mathcal{C}}, c \in \mathbb{R}\right.$,

$\nu \models \mathcal{I}(\ell)\}$. The initial state is $s_{0}^{\prime}=\left(\ell_{0}, \mathbf{0}, 0\right)$. We have $M_{1}^{\prime}=[\delta, \infty) \times E_{1}$, and $M_{2}^{\prime}=[\delta, \infty) \times E_{2} \cup[-\delta, \delta]$. The enabling conditions $T_{i}^{\prime}$ are as follows. For any $i \in\{1,2\}$, from any state $(\ell, \nu, c) \in S^{\prime}$, we have $((\ell, \nu, c), d, e) \in T_{i}^{\prime}$ for any $d \geq \delta$ and $e \in E_{i}$ whenever $(d, e)$ is enabled at $(\ell, \nu)$. We also have $(\ell, \nu, c), \perp) \in T_{i}^{\prime}$. For states $(\ell, \nu, c, d, e) \in S^{\prime}$, we have $((\ell, \nu, c, d, e), \perp) \in T_{1}^{\prime}$ and $((\ell, \nu, c, d, e), \epsilon) \in T_{2}^{\prime}$ for any $\epsilon \in[-\delta, \delta]$. The joint transition function $\delta$ respects the shorter delay:

$$
\mathrm{jt}^{\prime}\left((\ell, \nu, c),\left(d_{1}, e_{1}\right),\left(d_{2}, e_{2}\right)\right)= \begin{cases}\left(\ell, \nu, c, d_{1}, e_{1}\right) & \text { if } d_{1} \leq d_{2} \\ \left(\ell_{2},\left(\nu+d_{2}\right)\left[R^{\prime} \leftarrow 0\right], c+d_{2} \cdot \mathcal{S}(\ell)\right) & \text { if } d_{1}>d_{2}\end{cases}
$$

Moreover, in case one player plays $\perp$, we let jt ${ }^{\prime}\left((\ell, \nu, c),\left(d, e_{1}\right), \perp\right)=\left(\ell, \nu, c, d, e_{1}\right)$ and $\mathrm{jt}^{\prime}\left((\ell, \nu, c), \perp,\left(d^{\prime}, e_{2}\right)\right)=\left(\ell_{2},\left(\nu+d^{\prime}\right)\left[R^{\prime} \leftarrow 0\right], c+d^{\prime} \cdot \mathcal{S}(\ell)\right)$, as expected. Last, we let jt $\left.{ }^{\prime}\left(\ell, \nu, c, d, e_{1}\right), \perp, \epsilon\right)=\left(\ell_{1},(\nu+d+\epsilon)[R \leftarrow 0], c+(d+\epsilon) \cdot \mathcal{S}(\ell)\right)$. 
Thus, the cost variable grows with derivative $\mathcal{S}(\ell)$ at location $\ell$, and the sojourn time is either the delay suggested by Perturbator if it is shorter, or the delay suggested by Controller and perturbed by Perturbator otherwise. Notice that, in this semantics the guard of an edge that is taken need not be satisfied after a perturbation (hence the term excess).

We also consider another natural semantics for perturbation, which we call the $\delta$-conservative perturbation semantics and denote $\mathcal{G}_{\delta}^{c}(\mathcal{A})$. This semantics is defined similarly with the only difference that the enabling condition for Controller from states $(\ell, \nu, c)$ are defined as follows. From any state $(\ell, \nu, c) \in S^{\prime}$, we have $((\ell, \nu, c), d, e) \in T_{1}^{\prime}$ for any $d \geq \delta$ and $e \in E_{1}$ whenever $(d+\epsilon, e)$ is enabled at $(\ell, \nu)$ for every $\epsilon \in[-\delta, \delta]$. In other terms, Controller should only suggest delays and edges whose guards are enabled under any perturbation of the delay. Consequently, this semantics forbids equality constraints, since these are never enabled.

Example 2. Figure 2 explains the differences between our two perturbation semantics: Controller has to suggest a delay such that the resulting valuation does not end up in the grey area; Perturbator can then shift this delay by $[-\delta, \delta]$. In the conservative semantics, no new behaviors are added, because the final delay chosen by Perturbator will satisfy the guard; in the excess semantics, new behaviors may occur because neighboring regions can be reached.

Example 1 (Cont'd). We come back to the WTG of Fig. 1, to illustrate the differences between the two perturbed semantics. Under the excess-perturbation semantics, as in the exact case, Controller can suggest a delay of 1 and choose the edge from $\ell_{1}$ to $\ell_{2}$. The location $\ell_{5}$ can thus be avoided. Now, one can see that the move of Perturbator determines the next location to be visited: if Perturbator adds a positive perturbation (i.e. if the delay is in $[1,1+\delta]$ ), then only location $\ell_{3}$ is reachable. Conversely, a negative perturbation enables only location $\ell_{4}$. To maximize the cost, Perturbator will force the play to $\ell_{3}$, so Controller can only ensure a cost of $10+\Theta(\delta)$.

We now focus on the conservative semantics. The above strategy is no more valid since in this case, Controller can only suggest delays of at least $1+\delta$. Then Perturbator can force the play to $\ell_{5}$. Here, the cost of winning is $12+\Theta(\delta)$.
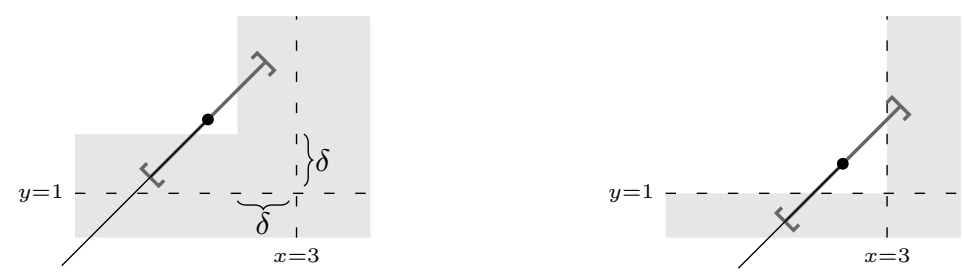

Fig. 2. The conservative- (left) and excess semantics (right) for a transition guarded with $x \leq 3 \wedge y \geq 1$. The grey area corresponds to forbidden delays, the bullet corresponds to the choice of Controller, and the segment indicates the possible choices for Perturbator. 
Cost-optimal reachability. We define cost-optimal reachability problems that take into account the perturbed semantics. We are interested in computing strategies for reachability which minimize the cost when the parameter $\delta$ goes to 0 .

First notice that $S_{1}\left(\mathcal{G}_{\delta}^{e}(\mathcal{A})\right)$ does not depend on $\delta$, since Controller only has to suggest moves that satisfy the guards (a winning strategy will depend on $\delta$ though). In contrast, $S_{1}\left(\mathcal{G}_{\delta}^{c}(\mathcal{A})\right)$ does depend on $\delta$ since Controller is required to satisfy the guards even after perturbations. It is easy to see that $S_{1}\left(\mathcal{G}_{\delta}^{c}(\mathcal{A})\right) \subseteq S_{1}\left(\mathcal{G}_{\delta^{\prime}}^{c}(\mathcal{A})\right)$ for any $\delta^{\prime}<\delta$. We denote $S_{1}\left(\mathcal{G}^{c}(\mathcal{A})\right)=\bigcup_{\delta>0} S_{1}\left(\mathcal{G}_{\delta}^{c}(\mathcal{A})\right)$.

Let us write $\left.(\ell, \nu, c)\right|_{\text {cost }}=c$, and $\left.(\ell, \nu, c, d, e)\right|_{\text {cost }}=c$, the projection of a state to the cost value. For any run $\rho$ of the exact or perturbed semantics, we define the cost of $\rho$ w.r.t. a location $\ell$ as, $\operatorname{cost}^{\ell}(\rho)=\inf \left\{\left.\operatorname{state}_{i}(\rho)\right|_{\text {cost }} \mid\right.$ $\left.1 \leq i<|\rho|+1, \operatorname{loc}\left(\operatorname{state}_{i}(\rho)\right)=\ell\right\}$ (Note that the definition includes the case where $|\rho|=\infty)$. Hence, if $\ell$ is never reached, then the cost is $\infty$. Otherwise it is the infimum of the costs observed at location $\ell$. Given $\delta>0$, a pair of strategies $\left(\sigma, \sigma^{\prime}\right) \in S_{1}\left(\mathcal{G}_{\delta}^{e}(\mathcal{A})\right) \times S_{2}\left(\mathcal{G}_{\delta}^{e}(\mathcal{A})\right)$, and location $\ell$, we define $\operatorname{cost}_{\sigma, \sigma^{\prime}}^{\ell}\left(\mathcal{G}_{\delta}^{e}(\mathcal{A})\right)=$ $\operatorname{cost}^{\ell}\left(\right.$ Outcome $\left._{\mathcal{G}_{\delta}^{e}(\mathcal{A})}\left(\sigma, \sigma^{\prime}\right)\right)$. We define similarly $\operatorname{cost}_{\sigma, \sigma^{\prime}}^{\ell}\left(\mathcal{G}_{\delta}^{c}(\mathcal{A})\right)$. Given a strategy $\sigma \in S_{1}\left(\mathcal{G}_{\delta}^{e}(\mathcal{A})\right.$, we define the limit-cost of $\sigma$ as $\lim ^{e} \operatorname{cost}_{\sigma}^{\text {exs }}(\mathcal{A}, \ell)=$ $\left.\lim _{\delta \rightarrow 0} \sup _{\sigma^{\prime} \in S_{2}\left(\mathcal{G}_{\delta}^{e}(\mathcal{A})\right)} \operatorname{cost}_{\sigma, \sigma^{\prime}}^{\ell}\left(\mathcal{G}_{\delta}^{e}(\mathcal{A})\right)\right)$. The limit is well defined since strategy $\sigma$ is valid for any $\delta>0$. Similarly, for $\sigma \in \mathcal{G}_{\delta_{0}}^{c}(\mathcal{A})$, we let $\lim$-cost ${ }_{\sigma}^{\text {cons }}(\mathcal{A}, \ell)=$ $\lim _{0<\delta<0<\delta_{0}} \sup _{\sigma^{\prime} \in S_{1}\left(\mathcal{G}_{\delta}^{c}(\mathcal{A})\right)} \operatorname{cost}_{\sigma, \sigma^{\prime}}^{\ell}\left(\mathcal{G}_{\delta}^{c}(\mathcal{A})\right)$. Here, we take the limit for $0<\delta<\delta_{0}$, such that $\sigma \in S_{2}\left(\mathcal{G}_{\delta_{0}}^{c}(\mathcal{A})\right)$ so that the strategy is valid for any considered $\delta$. Thus, the limit-cost of a Controller's strategy $\sigma$ is the cost it guarantees in the limit, against any strategy of Perturbator, when $\delta$ goes to 0 .

We are interested in deciding whether Controller has a strategy that guarantees an upper bound on the limit-cost for a reachability objective.

Definition 2. The limit strategy (strict) upper-bound problem for the excess perturbation semantics asks, given a weighted timed game $\mathcal{A}$, a location $\ell$, and a rational $\lambda$, whether there exists a strategy $\sigma \in S_{1}\left(\mathcal{G}_{\delta}^{e}(\mathcal{A})\right)$ such that lim-cost ${ }_{\sigma}^{\text {exs }}(\mathcal{A}, \ell) \leq \lambda$ (resp. lim-cost $\sigma_{\sigma}^{e x s}(\mathcal{A}, \ell)<\lambda$ ). Similarly, we define the limit strategy (strict) upperbound problem for the conservative perturbation semantics.

We define the limit-value as the infimum of the limit-cost that can be guar-

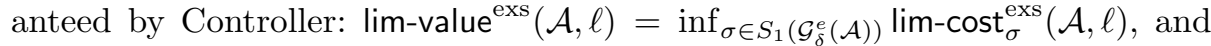
lim-value ${ }^{\text {cons }}(\mathcal{A}, \ell)=\inf _{\sigma \in S_{1}\left(\mathcal{G}^{c}(\mathcal{A})\right)} \lim$-cost ${ }_{\sigma}^{\text {cons }}(\mathcal{A}, \ell)$. We also consider deciding upper bounds on values:

Definition 3. The limit value upper-bound problem for the excess (resp. conservative) perturbation semantics asks whether given a weighted timed automaton $\mathcal{A}$, a target location $\ell$, and a rational $\lambda$, it holds lim-value ${ }^{\text {exs }}(\mathcal{A}, \ell) \leq \lambda$ (resp. $\lim$-value $\left.{ }^{\text {cons }}(\mathcal{A}, \ell) \leq \lambda\right)$ ?

Notice that the strategy strict upper-bound problem is equivalent to deciding whether the strict upper bound holds for the value, that is the infimum of the limit cost over all possible strategies. We will consider the restriction of both problems on WTA. 
Example 1 (Cont'd). We come back to the WTG of Fig. 1. We have seen the impact of the choice of the semantics on the possible behaviors of the system. In particular, the limit-optimal cost in the conservative (resp. excess) semantics is equal to 12 (resp. 10).

We now present our main results.

Theorem 1. The limit strategy upper-bound problem for WTA (and WTG) is undecidable under the excess perturbation semantics, for a fixed number of clocks.

Theorem 1 is a rather surprising result. It reveals that adding perturbations can render problems intractable, which is the opposite of a common belief [5, 17]. In this case, optimal reachability is PSPACE-complete for weighted timed automata under the exact semantics, but becomes undecidable under the excess perturbation semantics.

The conservative robust semantics is more restrictive than the excess perturbation semantics. In timed automata, reachability under the conservative semantics is PSPACE-complete [22], in contrast with the EXPTIME-completeness under the excess perturbation semantics [11]. For weighted timed automata, the conservative semantics renders the problem tractable; the limit value upper-bound problem is PSPACE-complete:

Theorem 2. The limit value upper-bound problem is PSPACE-complete on WTA under the conservative perturbation semantics.

The algorithm is based on the corner-point abstraction [8], but requires eliminating punctual regions, following the ideas in [22]. However the conservative semantics does not allow the treatment of weighted timed games:

Theorem 3. The limit strategy strict upper-bound problem is undecidable for WTG under the conservative perturbation semantics, for a fixed number of clocks.

The undecidability also holds in both semantics on WTGs when $\delta$ is fixed:

Theorem 4. The following problem is undecidable: For any fixed $0 \leq \delta \leq \frac{1}{3}$, given a WTG $\mathcal{A}$, a target location $\ell$, and a rational $\lambda$, decide whether it holds $\inf _{\sigma \in S_{1}(G)} \sup _{\sigma^{\prime} \in S_{2}(G)} \operatorname{cost}_{\sigma, \sigma^{\prime}}^{\ell}(G)<\lambda$, where $G$ denotes either $\mathcal{G}_{\delta}^{e}(\mathcal{A})$ or $\mathcal{G}_{\delta}^{c}(\mathcal{A})$.

In Section 4, we present our results on WTA, that is, the algorithm of Theorem 2 and the undecidability result of Theorem 1 . The technical appendix contains the detailed proofs of these results, and of Theorems 3 and 4 .

\section{Weighted Timed Automata}

\subsection{Algorithm in the Conservative Semantics}

We present a polynomial-space algorithm for the limit value upper-bound problem, based on a variant of the corner-point abstraction [8]. The idea behind our algorithm is that Perturbator can always avoid punctual regions by adding an infinitesimal perturbation. Thus, one needs to remove punctual delay transitions 
from the corner-point abstraction. It turns out that the resulting construction suffices to solve the limit-cost value for a given WTA.

A finite weighted automaton over $(\mathbb{Z},+)$ is a tuple $\mathcal{F}=\left(S, s_{0}, \Sigma, T, W\right)$, where $S$ is the set of states, $s_{0} \in S$ the initial state, $T \subseteq S \times \Sigma \times S$ the set of transitions, and $W: E \rightarrow \mathbb{Z}$ is the weight function. A path (or run) of a finite weighted automaton is a (finite or infinite) sequence $q_{1} t_{1} q_{2} t_{2} \ldots$ alternating states and transitions and such that for all $i \geq 1, t_{i}=\left(q_{i}, \sigma_{i}, q_{i+1}\right)$ for some $\sigma_{i}$. We write $\operatorname{Runs}(\mathcal{F})$ for the set of runs of $\mathcal{F}$ starting in the initial state $s_{0}$. The length, first and last states and sub-runs are defined in the same way as for runs of a game structure. A finite weighted automaton then associates to any finite path the sum of the weights of the edges it visits. Given any path $\pi=q_{1} t_{1} q_{2} \ldots q_{n}$, the weight of $\pi$ is defined as $W(\pi)=\sum_{1 \leq i<n} W\left(t_{i}\right)$.

Let us consider a weighted timed automaton $\mathcal{A}=\left(\mathcal{L}, \ell_{0}, \mathcal{C}, E_{1}, \emptyset, \mathcal{S}\right)$. Notice that, following Def. 1 , we write it as a weighted timed games with no edges belonging to Player 2. The corner-point abstraction of $\mathcal{A}$ is a finite weighted automaton, denoted $\mathcal{R}_{\mathrm{cp}}(\mathcal{A})$. The states of $\mathcal{R}_{\mathrm{cp}}(\mathcal{A})$ are triples $(\ell, r, v)$, where $\ell$ is a location, $r$ a region, and $v \in \mathcal{V}(r)$ a vertex of $r$. Edges are defined as follows: we have $(\ell, r, v) \stackrel{\text { delay }}{\longrightarrow}\left(\ell, r^{\prime}, v^{\prime}\right)$ if $(\ell, r) \stackrel{\text { delay }}{\longrightarrow}\left(\ell, r^{\prime}\right)$ in the region automaton, and $v^{\prime}=v+k$ for some natural number $k$. In other terms, $v^{\prime}$ is a time-successor of $v$, and is a vertex of region $r^{\prime}$. The weight associated to this transition is $k \times \mathcal{S}(\ell)$. Further, we have an edge $(\ell, r, v) \stackrel{e}{\rightarrow}\left(\ell^{\prime}, r^{\prime}, v^{\prime}\right)$ if $e=\left(\ell, g, \sigma, R, \ell^{\prime}\right)$ is an edge of $\mathcal{A}$ such that $r=g$, and $r^{\prime}=r[R \leftarrow 0], v^{\prime}=v[R \leftarrow 0]$. Such an edge has weight 0 . Observe that $\mathcal{R}_{\mathrm{cp}}(\mathcal{A})$ is finite since all clocks are assumed to be bounded. Notice that a path in the corner-point abstraction corresponds to a path of the WTA that runs arbitrarily close to vertices of the regions it visits.

Let the non-punctual corner-point abstraction, denoted $\mathcal{R}_{\mathrm{cp}}^{\mathrm{np}}(\mathcal{A})$, be the finite weighted automaton obtained from the corner-point abstraction by removing any transition of the form $(\ell, r, v) \stackrel{\text { delay }}{\longrightarrow}\left(\ell, r^{\prime}, v^{\prime}\right)$, where $r^{\prime}$ is punctual. Thus, any path in the non-punctual corner-point abstraction corresponds to a non-punctual path in the region automaton.

For any path $\pi$ of the region automaton $\mathcal{R}(\mathcal{A})$ of $\mathcal{A}$, we denote by $\operatorname{Runs}(\pi)$, the set of runs of $\llbracket \mathcal{A} \rrbracket$ that follow $\pi$. If $\pi$ is a path of the corner-point abstraction $\mathcal{R}_{\mathrm{cp}}(\mathcal{A})$, then we say that a run follows $\pi$ if it follows the path projected to $\mathcal{R}(\mathcal{A})$ (that is, obtained by removing vertices in each state). We extend the notation $\operatorname{Runs}(\pi)$ to paths $\pi$ of the corner-point abstraction. For any path $\pi$ of $\mathcal{R}(\mathcal{A})$ or $\mathcal{R}_{\text {cp }}(\mathcal{A})$, let us define $\bar{\pi}$ obtained from $\pi$ by replacing all regions by their topological closures. We will consider Runs $(\bar{\pi})$ which is the set of runs visiting the topological closures of the regions of $\pi$. In other terms, this is the topological closure of the set $\operatorname{Runs}(\pi)$.

We define value $(\mathcal{F}, s)$ for a finite weighted automaton $\mathcal{F}$ and state $s$ as the cost of the shortest path from the initial state to $s$. Formally, for any finite weighted automaton $\mathcal{F}=\left(S, s_{0}, \Sigma, T, W\right)$, and $s \in S$, we let value $(\mathcal{F}, s)=\inf \{W(\pi) \mid \pi \in$ $\operatorname{Runs}(\mathcal{F})$, last $(\pi)=s\}$. For corner-point abstractions, we extend this notation to locations: $\operatorname{value}\left(\mathcal{R}_{\mathrm{cp}}(\mathcal{A}), \ell\right)=\inf \left\{W(\pi) \mid \pi \in \operatorname{Runs}\left(\mathcal{R}_{\mathrm{cp}}(\mathcal{A})\right), \operatorname{loc}(\right.$ last $\left.(\pi))=\ell\right\}$. 
In the exact semantics, results of [8] show that the infimum of the cost of the runs of a WTA following a given path $\pi$ of the corner-point abstraction is achieved by a run that follows $\bar{\pi}$, and only visits vertices. Hence, to compute the infimum cost, it suffices to compute the value of the corner-point abstraction. In the conservative perturbed case, we prove that the same algorithm can be applied, once we discard punctual paths.

Lemma 1. For any weighted timed automaton $\mathcal{A}$ and target location $\ell$, we have $\lim$-value ${ }^{\text {cons }}(\mathcal{A}, \ell)=\operatorname{value}\left(\mathcal{R}_{c p}^{n p}(\mathcal{A}), \ell\right)$.

Theorem 2 follows from the previous lemma. In fact, to compute the optimal cost on $\mathcal{A}$, it suffices to consider the finite weighted automaton $\mathcal{R}_{\mathrm{cp}}^{\mathrm{np}}(\mathcal{A})$, and find the shortest path to location $\ell$. To decide whether the limit value is less than some given constant $\lambda$, one can guess a path in $\mathcal{R}_{\mathrm{cp}}^{\mathrm{np}}(\mathcal{A})$ in polynomial-space (such a path can be constructed on-the-fly in polynomial space, see e.g. [8]), and check whether its weight is less than or equal to $\lambda$. Note that the problem is PSPACE-hard since it already is in the unweighted case.

\subsection{Undecidability Under Excess Perturbation}

In this section, we present the proof of Theorem 1, showing the undecidability of the limit strategy upper-bound problem for WTA with excess perturbation. Our proof is based on a reduction from the halting problem of Minsky machines, following the encoding of [9]. Compared to the reductions of earlier work [12, 9], special care needs to be taken when dealing with perturbations, since the present semantics disables precise moves.

We consider a Minsky machine with counters $c_{1}$ and $c_{2}$, and a list of instructions $I_{1}, \ldots, I_{n}$. Here, each instruction $I_{i}$, for $1 \leq i \leq n-1$, is an incrementation for $c_{b}$ as, $c_{b}=c_{b}+1$; goto $I_{j}$, for $b=1$ or 2 , or a decrementation with zerotest for $c_{b}$ as, if $\left(c_{b}=0\right)$ goto $I_{j}$ else $c_{i}=c_{i}-1$; goto $I_{j^{\prime}}$. The instruction $I_{n}$ is the ending instruction, that is, the final state. The halting problem asks whether the instruction $I_{n}$ is reachable, starting from the configuration $c_{1}=0$, $c_{2}=0$, at instruction $I_{1}$.

Our reduction uses 10 clocks $x, x^{\prime}, y, y^{\prime}, u, u^{\prime}, t, t^{\prime}, z, z^{\prime}$. A counter of a Minsky machine with value $n$ will be encoded by a pair of clocks $x, x^{\prime}$ with values $k_{x}+\frac{1}{2^{n}}$ and $k_{x^{\prime}}+\frac{1}{2^{n}}$ for some integers $k_{x}, k_{x^{\prime}}$. Here, $k_{x}$ is called the shift of $x$. If $\alpha$ denotes the clock $x^{\prime}$, we let $\alpha^{\prime}=x$, and $\alpha^{\prime \prime}=x^{\prime}$, and similarly for other clocks. A configuration of a Minsky machine with counter values $n, m \geq 0$, is entirely encoded by four clocks:

$$
x=k_{x}+\frac{1}{2^{n}} \quad x^{\prime}=k_{x^{\prime}}+\frac{1}{2^{n}} \quad y=k_{y}+\frac{1}{2^{m}} \quad y^{\prime}=k_{y^{\prime}}+\frac{1}{2^{m}}
$$

for some shifts $k_{x}, k_{x^{\prime}}, k_{y}, k_{y^{\prime}}$. The redundancy in this encoding is necessary to cope with perturbations; this will be clear in the constructions. We denote by $\boldsymbol{k}$ the vector of shifts, for all clocks, and by $\operatorname{code}_{\boldsymbol{k}}(n, m)$ the set of valuations satisfying (1). We also define $\operatorname{code}_{\boldsymbol{k}}^{\epsilon}(n, m)$ the set of valuations $\nu$ such that $\nu+\eta \in \operatorname{code}_{\boldsymbol{k}}(n, m)$, where $|\eta(\alpha)| \leq \epsilon, \eta(\alpha)=\eta\left(\alpha^{\prime}\right)$ for all clocks $\alpha$, and moreover 


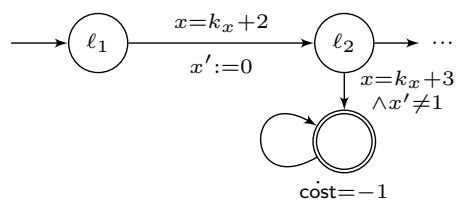

(a) An unperturbed edge.

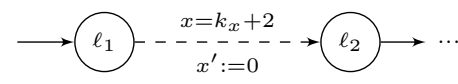

(b) A simpler representation of that edge.

Fig. 3. Unperturbed edges. Perturbator has interest in not perturbing these transitions, since otherwise Controller can go to the target location and win with cost $-\infty$.

$\eta(x)=\eta\left(x^{\prime}\right)=0$ whenever $n=0$, and $\eta(y)=\eta\left(y^{\prime}\right)=0$ whenever $m=0$. In other terms, $\operatorname{code}_{k}^{\epsilon}(n, m)$ is the set of valuations that encode a configuration with an error bounded by $\epsilon$, except that the encoding of the counter value 0 is exact. Given shifts $\boldsymbol{k}$ and a valuation $\nu \in \operatorname{code}_{\boldsymbol{k}}^{\epsilon}(n, m)$, we denote $\operatorname{fracp}(\nu)=\nu-\boldsymbol{k}$. This gives the fractional part of the clocks, except when $n=0$, in which case the components $x$ and $x^{\prime}$ are equal to 1 , and similarly for $y$. We say that a valuation $\nu$ encodes a configuration $(n, m)$ of the machine if it satisfies (1) for some $\boldsymbol{k}$.

We define modules for incrementation and decrementation with zero-test instructions, which will, once combined, yield the reduction. The modules will be defined on a given list of clocks. For instance, if we describe a module $M(x, y, z, u, t)$ that uses the clocks $x, y, z, u$, and $t$ in its definition, then $M(z, y, x, t, u)$ is obtained simply by exchanging $x$ and $z$, and $u$ and $t$.

Unperturbed edges. Let us first present a construction that prevents Perturbator from perturbing the delays along an edge. The construction only applies to deterministic transitions (with equality constraints) and requires resetting one of the two clocks used in the encoding of a counter. Consider the timed automaton of Fig. 3(a). At $\ell_{2}$, Controller can go to the accepting state where the cost decreases to $-\infty$ if, and only if Perturbator has perturbed by a nonzero amount the transition from $\ell_{1}$ to $\ell_{2}$. Thus, Perturbator does not have interest in perturbing since its objective is to maximize the cost. If there has been no perturbation, the clock values are only increased or decreased by some integers. More precisely, the shifts of all clocks but $x^{\prime}$ increase by 2 , and the shift of $x^{\prime}$ becomes 0 . In the rest, we will use this trick extensively to construct our modules. For better readability, we will represent such unperturbed edges by dashed arrows; when clear from context, we may omit the edges leading to accepting sink states (see Fig. 3(b)).

Ask-Perturbator module. In weighted timed automata, unlike in weighted timed games, Perturbator cannot suggest moves since it controls no edges. However, a special construction allows letting Perturbator decide the successor location. We describe in Fig. 4(a) such a construction, which also ensures that the configuration is preserved, up to shifts.

The first edge is deterministic, and Perturbator can add any perturbation. Controller then distinguishes between positive and negative perturbations, and only has one possible move accordingly. We disallow perturbations (using unperturbed edges) at the edges leading to $\ell_{2}$ or $\ell_{3}$, so that the configuration is preserved up to shifts. More precisely, the shifts of all clocks $x, x^{\prime}, y, y^{\prime}$ increase 


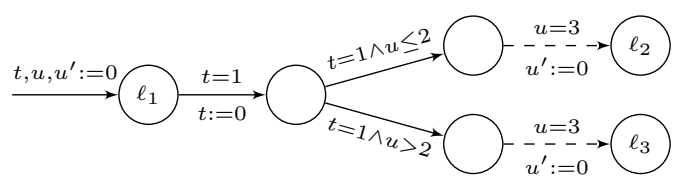

(a) Letting Perturbator decide.

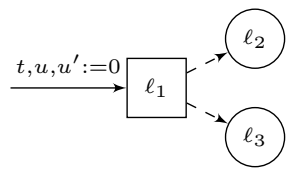

(b) A simpler representation of the automaton opposite.

Fig. 4. Module that lets Perturbator decide a successor among $\ell_{2}$ and $\ell_{3}$.

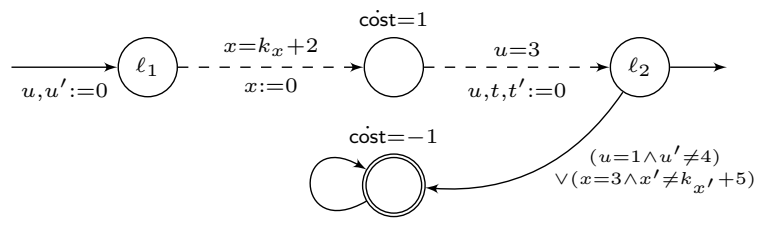

Fig. 5. Module $\operatorname{Add}_{\boldsymbol{k}}^{1+x}(x, u, t)$.

by 3 . To simplify the presentation of more complex modules, we will represent this module more compactly as in Fig. 4(b).

Reduction module. In the above modules, we have seen that configurations are preserved up to shifts, but shifts could grow. We present a module that reduces the shifts of all clocks. The module $\operatorname{Reduce}_{\boldsymbol{k}}(x, y, u, t)$ is constructed for each shift vector $\boldsymbol{k}$ (there will be a finite number of these), is deterministic, and constructed using unperturbed edges. The definition of the module is omitted (see Appendix B); the following lemma summarizes its properties.

Lemma 2. Let $\delta<\frac{1}{2}$. Assume $\operatorname{Reduce}_{\boldsymbol{k}}(x, y, u, t)$ is entered with valuation $\nu \in$ $\operatorname{code}_{\boldsymbol{k}}^{\epsilon}(n, m)$ for some $\epsilon<\frac{1}{2}$. Controller has a strategy to either go to the target location with cost $-\infty$ or to reach location $\ell_{2}$ with valuation $\nu^{\prime}$ satisfying $\nu^{\prime}=$ $\operatorname{fracp}(\nu)+\boldsymbol{k}^{\prime}$ where $\boldsymbol{k}^{\prime}$ is defined as follows: $k_{x}^{\prime}=6, k_{x^{\prime}}^{\prime}=2, k_{y}^{\prime}=5, k_{y^{\prime}}^{\prime}=1$.

Test Module. In order to verify the incrementation and decrementation, we will use the cost variable. We first show how one can add $1+\operatorname{fracp}(x)$ and $2-\operatorname{fracp}(x)$ to the cost variable, without changing the configuration. The construction is similar to [9]; we adapt it using unperturbed edges.

The module $\operatorname{Add}_{\boldsymbol{k}}^{1+x}(x, u, t)$ depicted in Fig. 5 adds $1+\operatorname{fracp}(x)$ to the cost, leaving the configuration unchanged (up to shifts).

Lemma 3. Let $\delta<\frac{1}{2}$. Assume module $\operatorname{Add}_{\boldsymbol{k}}^{1+x}(x, u, t)$ is entered with valuation $\nu \in \operatorname{code}_{\boldsymbol{k}}^{\epsilon}(n, m)$ for some $\epsilon<\frac{1}{2}$. Controller has a strategy that ensures either reaching a target location with cost $-\infty$ or location $\ell_{2}$ with valuation $\nu^{\prime}$ satisfying $\nu^{\prime}=\operatorname{fracp}(\nu)+\boldsymbol{k}^{\prime}$ where $k_{x}^{\prime}=1$, and $k_{\alpha}^{\prime}=k_{\alpha}+3$ for all $\alpha \in\left\{x^{\prime}, y, y^{\prime}, z, z^{\prime}\right\}$, while the cost increases by $1+\operatorname{fracp}(x)$.

We define similarly a module $\operatorname{Add}_{k}^{2-x}(x, u, t)$ that adds $2-\operatorname{fracp}(x)$ to the cost variable. The module is similar to the one of Fig. 5, except that cost only increases (with slope 1 ) at location $\ell_{1}$. 
Lemma 4. Let $\delta<\frac{1}{2}$. Assume module $\operatorname{Add}_{\boldsymbol{k}}^{2-x}(x, u, t)$ is entered with valuation $\nu \in \operatorname{code}_{\boldsymbol{k}}^{\epsilon}(n, m)$ for some $\epsilon<\frac{1}{2}$. Controller has a strategy that ensures either reaching a target location with cost $-\infty$ or location $\ell_{2}$ with valuation $\nu^{\prime}$ satisfying $\nu^{\prime}=\operatorname{fracp}(\nu)+\boldsymbol{k}^{\prime}$ where $k_{x}^{\prime}=1$, and $k_{\alpha}^{\prime}=k_{\alpha}+3$ for all $\alpha \in\left\{x^{\prime}, y, y^{\prime}, z, z^{\prime}\right\}$, while the cost increases by $2-\operatorname{fracp}(x)$.

Concatenating modules $\operatorname{Add}_{k}^{1+x}(x, u, t), \operatorname{Add}_{k^{\prime}}^{2-x}(z, t, u)$ and $\operatorname{Add}_{k^{\prime \prime}}^{2-x}(z, u, t)$, we obtain the module $\operatorname{Add}_{\boldsymbol{k}}^{5+x-2 z}(x, z, u, t)$, which adds $5+\operatorname{fracp}(x)-2 \operatorname{fracp}(z)$ to the cost and leads to a target location (we make the last location accepting). Similarly, using concatenation, we define $\operatorname{Add}_{k}^{4+2 z-x}(x, z, u, t)$, which increases the cost by $4+2 \operatorname{fracp}(z)-\operatorname{fracp}(x)$. One can append at the end of module $\operatorname{Add}_{k}^{4+2 z-x}(x, z, u, t)$ an edge that increments the cost by 1 , which yields module $\operatorname{Add}_{k}^{5+2 z-x}(x, z, u, t)$ (see Fig. 8). Finally, module Test ${ }^{2 z=x}(x, z, u, t)$ is defined by letting Perturbator choose whether to go to $\operatorname{Add}_{\boldsymbol{k}^{\prime}}^{5+2 z-x}(x, z, t, u)$ or to $\operatorname{Add}_{\boldsymbol{k}^{\prime}}^{5+x-2 z}(x, z, t, u)$ (here the new shift vector $\boldsymbol{k}^{\prime}$ is due to the shift added by the ask-Perturbator module). Note that in both cases, the run ends in a target location. The following property follows from Lemmas 3 and 4.

Lemma 5. Let $\delta<\frac{1}{2}$. If module Test ${ }^{2 z=x}(x, z, u, t)$ is entered with valuation $\nu \in$ $\operatorname{code}_{\boldsymbol{k}}^{\epsilon}(n, m)$ for some $\epsilon<\frac{1}{2}$, Controller has a strategy to ensure reaching a target location with cost at most $5+|2 \operatorname{fracp}(z)-\operatorname{fracp}(x)|$.

Incrementation Module Aut ${ }_{k}{ }^{c_{1},+}$. We define module Aut $_{k}{ }^{c_{1},+}$, given in Fig. 6, which simulates the incrementation of counter $c_{1}$. We assume first that there are no perturbation. When the module is entered with a valuation in $\operatorname{code}_{\boldsymbol{k}}(n, m)$, we expect Controller to choose the delays so that $z=1+\frac{\operatorname{fracp}(x)}{2}$ at location $D$. From this point on, the clocks $z$ and $z^{\prime}$ will switch roles with $x$ and $x^{\prime}$. Thus, this corresponds to incrementing the counter $c_{1}$ by 1 . At location $D$, Perturbator can either decide to "test" the incrementation has been correctly performed by going to the test module, or to continue the simulation by first passing through the reduction module. Here, $\operatorname{Instr}_{\boldsymbol{k}^{\prime \prime}}^{j}$ refers to a module among $\mathrm{Aut}_{\boldsymbol{k}}{ }^{c_{b},+}$, Aut $_{k}{ }^{c_{b},-}$ for $b \in\{1,2\}$ (to be defined next) according to the instruction $I_{j}$. Now, in the presence of perturbations, Perturbator can perturb the value of $z$ chosen by Controller by $\delta$. So at $D$, if Perturbator goes to the test module the cost is $5+O(\delta)$, provided that Controller has played correctly. Otherwise, the simulation carries on with $\left|z-\frac{\operatorname{fracp}(x)}{2}\right| \leq \delta$. The following lemma states this formally.

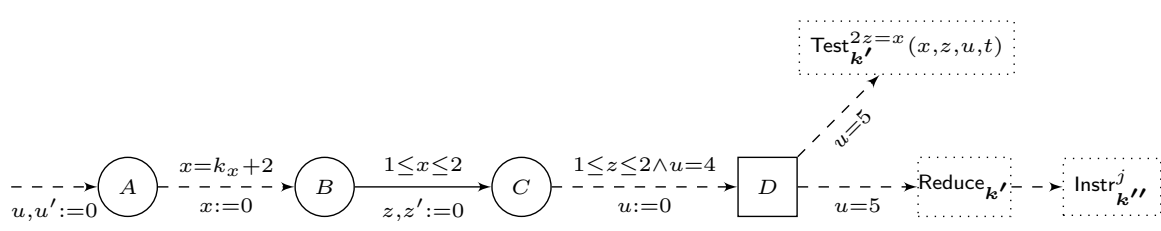

Fig. 6. Module $\operatorname{Aut}_{\boldsymbol{k}}{ }^{c_{1},+}(x, y, z, u, t)$. The module $\operatorname{Reduce}_{\boldsymbol{k}^{\prime}} \operatorname{refers}$ to $\operatorname{Reduce}_{\boldsymbol{k}^{\prime}}(z, y, u, t)$, and the module $\operatorname{Instr}_{\boldsymbol{k}^{\prime \prime}}^{j}$ to $\operatorname{Instr}_{\boldsymbol{k}^{\prime \prime}}^{j}(z, y, x, u, t)$. 
Lemma 6. Let $\delta<\frac{1}{2}$. Assume module Aut ${ }_{\boldsymbol{k}}^{c_{1},+}$ is entered with valuation $\nu \in$ $\operatorname{code}_{\boldsymbol{k}}^{\epsilon}(n, m)$ for some $\epsilon>0$ with $\delta+\epsilon / 2<\frac{1}{2}$, and cost 0 . Then, Controller has a strategy that ensures that either module $\operatorname{Instr}_{k}{ }^{j}$ is entered with a valuation $\nu^{\prime} \in \operatorname{code}_{\boldsymbol{k}^{\prime}}^{\delta+\epsilon / 2}(n+1, m)$ for some $\boldsymbol{k}^{\prime}$, or the target location is reached with cost at most $5+2 \delta$.

A decrementation module can be defined following the same ideas.

Lemma 7. Assume module Aut $_{\boldsymbol{k}}{ }^{c_{1},-}$ is entered with valuation $\nu \in \operatorname{code}_{\boldsymbol{k}}^{\epsilon}(n, m)$ and cost 0 . Then,

- If $n=0$, Controller has a strategy to ensure reaching either Instr $_{k}{ }^{j}$ with the same configuration up to shifts and cost, or an accepting location with cost 0 .

- If $n \geq 1$, and $\epsilon<\frac{1}{2}$, the play cannot reach $\operatorname{Instr}_{k}{ }^{j}$.

- If $n \geq 1$, Controller has a strategy that ensures that either module Instr $_{k}{ }^{j^{\prime}}$ is reached with a valuation $\nu^{\prime} \in \operatorname{code}_{\boldsymbol{k}^{\prime}}^{\delta+2 \epsilon}(n-1, m)$ for some $\boldsymbol{k}^{\prime}$, or the target location is reached with cost at most $5+\epsilon+2 \delta$. Moreover, if $n=1$, then Controller can ensure that $\nu^{\prime}(x)=k_{x}^{\prime}+1$.

Complete reduction. To construct the complete reduction, we define for each instruction $I_{j}$ of the Minsky machine, a module $\operatorname{Instr}_{k}{ }^{j}$ as one of the incrementation or decrementation modules according to the type of $I_{i}$. We mark the first location of $\operatorname{Instr}_{k}{ }^{1}$ as the initial location. The halting state $\operatorname{Instr}_{k}{ }^{n}$ of the machine is an accepting location of the timed automaton. For any machine $M$, let $\mathcal{A}_{M}$ denote the weighted timed automaton constructed in this manner, and let $\ell$ denote the target location obtained by merging all target locations presented in the above construction. Theorem 1 follows from the following proposition.

Proposition 1. The Minsky machine $M$ halts if, and only if, there is a strategy $\sigma \in S_{C}\left(\mathcal{G}^{\text {exs }}\left(\mathcal{A}_{M}\right)\right)$ such that lim-cost $\operatorname{exs}_{\sigma}\left(\mathcal{A}_{M}, \ell\right) \leq 5$.

Discussion. One can argue that the undecidability in the excess perturbation game semantics is due to the ability of Controller to test clock values with precision using equality constraints, and in particular in detecting perturbations. This allows for instance letting Perturbator make a discrete choice, as in the above reduction. Hence, this ability and the possibility of disallowing perturbations on some edges make the semantics of weighted timed automata somehow close to that of two-player weighted timed games in the exact semantics for which the optimal-cost reachability is undecidable.

The conservative perturbation game semantics disallows both abilities since Controller is required to suggest delays whose perturbations satisfy the guard of the chosen edge. This excludes equality constraints from guards. Therefore, one cannot encode unperturbed edges nor define the ask-Perturbator module as previously. The decidability proof presented in Section 4 confirms these intuitions.

\section{Conclusion}

In this paper, we showed "robust undecidability" results for weighted timed games: optimal reachability problems remain undecidable under perturbation 
game semantics. Moreover, the problem even becomes undecidable for weighted timed automata in the excess perturbation game semantics. The undecidability in both cases is due to the ability of either of the players to play precisely, and the other one to check previous delays with precision. We conclude that game semantics does not introduce enough "fuzziness" in the semantics to avoid encoding undecidable languages.

We did not study the value upper-bound problems for the excess perturbation game semantics; we conjecture that it should be undecidable. We believe we could also recover decidability by restricting to closed guards, since then players would not be able to check the non-equality of the clock values.

\section{References}

1. R. Alur, M. Bernadsky, and P. Madhusudan. Optimal reachability for weighted timed games. In ICALP'04, LNCS, p. 122-133. Springer, 2004.

2. R. Alur and D. L. Dill. A theory of timed automata. Theoretical Computer Science, 126(2):183-235, 1994.

3. R. Alur, S. La Torre, and P. Madhusudan. Perturbed timed automata. In HSCC'05, LNCS 3414, p. 70-85. Springer, 2005.

4. R. Alur, S. La Torre, and G. J. Pappas. Optimal paths in weighted timed automata. In HSCC'01, LNCS 2034, p. 49-62. Springer, 2001.

5. E. Asarin and A. Bouajjani. Perturbed Turing machines and hybrid systems. In LICS'01, p. 269-278. IEEE Comp. Soc. Press, 2001.

6. E. Asarin, O. Maler, A. Pnueli, and J. Sifakis. Controller synthesis for timed automata. In SSC'98, p. 469-474. Elsevier Science, 1998.

7. G. Behrmann, A. Fehnker, T. Hune, K. G. Larsen, P. Pettersson, J. Romijn, and F. Vaandrager. Minimum-cost reachability for priced timed automata. In HSCC'01, LNCS 2034, p. 147-161. Springer, 2001.

8. P. Bouyer, Th. Brihaye, V. Bruyère, and J.-F. Raskin. On the optimal reachability problem of weighted timed automata. Formal Methods in System Design, 31(2):135$175,2007$.

9. P. Bouyer, Th. Brihaye, and N. Markey. Improved undecidability results on weighted timed automata. Information Processing Letters, 98(5):188-194, 2006.

10. P. Bouyer, F. Cassez, E. Fleury, and K. G. Larsen. Optimal strategies in priced timed game automata. In FSTTCS'04, LNCS 3328, p. 148-160. Springer, 2004.

11. P. Bouyer, N. Markey, and O. Sankur. Robust reachability in timed automata: a game-based approach. In ICALP'12, LNCS 7392, p. 128-140. Springer, 2012.

12. Th. Brihaye, V. Bruyère, and J.-F. Raskin. On optimal timed strategies. In FORMATS'05, LNCS 3829, p. 49-64. Springer, 2005.

13. K. Chatterjee, T. A. Henzinger, and N. Piterman. Strategy logic. Information and Computation, 208(6):677-693, 2010.

14. K. Chatterjee, T. A. Henzinger, and V. S. Prabhu. Timed parity games: Complexity and robustness. Logicical Methods in Computer Science, 7(4), 2011.

15. M. De Wulf, L. Doyen, N. Markey, and J.-F. Raskin. Robust safety of timed automata. Formal Methods in System Design, 33(1-3):45-84, 2008.

16. M. De Wulf, L. Doyen, and J.-F. Raskin. Almost ASAP semantics: From timed models to timed implementations. Formal Aspects of Computing, 17(3):319-341, 2005.

17. M. Fränzle. Analysis of hybrid systems: an ounce of realism can save an infinity of states. In CSL'99, LNCS 1862, p. 126-139. Springer, 1999. 
18. V. Gupta, T. A. Henzinger, and R. Jagadeesan. Robust timed automata. In HART'97, LNCS 1201, p. 331-345. Springer, 1997.

19. T. A. Henzinger and J.-F. Raskin. Robust indecidability of timed and hybrid systems. In HSCC'00, LNCS 1790, p. 145-159. Springer, 2000.

20. A. Puri. Dynamical properties of timed systems. Discrete Event Dynamic Systems, 10(1-2):87-113, 2000.

21. O. Sankur, P. Bouyer, and N. Markey. Shrinking timed automata. In FSTTCS'11, LIPIcs 13, p. 375-386. Leibniz-Zentrum für Informatik, 2011.

22. O. Sankur, P. Bouyer, N. Markey, and P.-A. Reynier. Robust controller synthesis in timed automata. In submission, 2013. 


\section{A Details of Subsection 4.1}

To prove Theorem 2, we first need to introduce some definitions.

A timed trace is a sequence $\left(t_{i}, e_{i}\right)_{1 \leq i \leq n}$ where $t_{i} \geq 0$ and each $e_{i}$ is an edge. The timed trace of a run $\rho$ is the sequence trace $(\rho)=\left(t_{i}, e_{i}\right)_{1 \leq i \leq n}$, where the $t_{i} \geq 0$ are the delay transitions of $\rho$, and $e_{i}$ are the edges it takes. Given a path $\pi$ of $\mathcal{R}(\mathcal{A})$, and state $(\ell, \nu) \in$ first $(\pi)$, we say that a timed trace $\left(t_{i}, e_{i}\right)_{1 \leq i \leq n}$ is feasible for $\pi$ and $(\ell, \nu)$ if there is a run starting at $(\ell, \nu)$, following $\pi$, whose timed trace is $\left(t_{i}, e_{i}\right)_{1 \leq i \leq n}$. We write untime $\left(\left(t_{i}, e_{i}\right)_{1 \leq i \leq n}\right)=\left(e_{i}\right)_{1 \leq i \leq n}$.

We define a metric on timed traces as in [18]. Given two timed traces $u=$ $\left(t_{i}, e_{i}\right)_{1 \leq i \leq n}$ and $u^{\prime}=\left(t_{i}^{\prime}, e_{i}^{\prime}\right)_{1 \leq i \leq n}$, we let

$$
\begin{aligned}
& d\left(u, u^{\prime}\right)=\infty \text { if untime }(u) \neq \text { untime }\left(u^{\prime}\right), \\
& d\left(u, u^{\prime}\right)=\max \left\{\left|t_{i}-t_{i}^{\prime}\right|, 1 \leq i \leq n\right\} \text { otherwise. }
\end{aligned}
$$

We define $\operatorname{Ball}_{d}(u, \epsilon)$ as the open ball of radius $\epsilon$ around $u$ in this metric. Note that the original metric was defined using timestamps rather than delays, but both metrics define the same topology [18].

For a path $\pi$, let $\operatorname{ttrace}(\pi)$ denote the set of timed traces that are feasible for $\pi$ and some state in first $(\pi)$.

Proposition 2 ([18]). If $\pi$ is a non-punctual path, then ttrace $(\pi)$ is an open set (for the topology induced by $d$ ).

The previous proposition follows from the fact that a non-punctual path can be described by an open timed automaton, that is, a timed automaton whose guards are strict.

We recall the following result on optimal cost reachability in the exact semantics. Let us write $\mathcal{V}((\ell, r))=\{\ell\} \times \mathcal{V}(r)$, for any location $\ell$ and region $r$.

Lemma 8 ([8]). Let $\mathcal{A}=\left(\mathcal{L}, \ell_{0}, \mathcal{C}, \mathcal{I}, E_{1}, \emptyset, \mathcal{S}\right)$ be a WTA, and $\pi$ a path in $\mathcal{R}(\mathcal{A})$. There exists a run $\rho_{0}$ that follows $\bar{\pi}$, such that for all $1 \leq i \leq|\rho|$, $\operatorname{state}_{i}(\rho) \in$ $\mathcal{V}\left(\operatorname{state}_{i}(\pi)\right)$, and

$$
\left.\operatorname{last}\left(\rho_{0}\right)\right|_{\text {cost }}=\inf _{\rho \in \operatorname{Runs}(\pi)}\left(\text { last }\left.(\rho)\right|_{\text {cost }}\right) .
$$

The previous lemma states that the infimum of the cost of the runs that follow a path of the region automaton is reached, in the limit, by a run that only visits the vertices of the regions. Observe that such a run corresponds to a path of the corner-point abstraction.

The following simple lemma states that an infinitesimal perturbation suffices to go from a punctual region to a non-punctual one.

Lemma 9. Given any $\delta>0$, and $\nu \in r$ in a punctual region $r$, there exists $\epsilon \in[-\delta, \delta]$ such that $\operatorname{reg}(\nu+\epsilon)$ is non-punctual.

We now prove Lemma 1:

Lemma 1. For any weighted timed automaton $\mathcal{A}$ and target location $\ell$, we have $\lim$-value ${ }^{\text {cons }}(\mathcal{A}, \ell)=\operatorname{value}\left(\mathcal{R}_{c p}^{n p}(\mathcal{A}), \ell\right)$. 
Proof. We prove the two inequalities. First, it is not difficult to show that $\lim$-value ${ }^{\text {cons }}(\mathcal{A}, \ell) \geq \operatorname{value}\left(\mathcal{R}_{\mathrm{cp}}^{\text {np }}(\mathcal{A}), \ell\right)$. In fact, let $\sigma \in S_{1}\left(\mathcal{G}_{\delta}^{c}(\mathcal{A})\right)$ be any strategy for Controller. We consider some strategy $\sigma^{\prime}$ for Perturbator, that perturbs so as to render the run non-punctual. More precisely, if the delay suggested by Controller is inside a non-punctual region, Perturbator plays 0, and otherwise it plays some small non-zero amount, by Lemma 9 . Then the projection of the outcome of $\left(\sigma, \sigma^{\prime}\right)$ to regions is a non-punctual path $\pi$ of $\mathcal{R}(\mathcal{A})$. But by Lemma 8, the cost of this run cannot be smaller than all runs along $\bar{\pi}$ visiting vertices, and for any such run, there is a path in $\mathcal{R}_{\mathrm{cp}}^{\mathrm{np}}(\mathcal{A})$ with the same cost.

To prove the other direction, we make use of the following lemma, which says that the paths of the corner-point abstraction can be approximated by valid runs.

Lemma $10([8])$. Let $\pi$ be a path of $\mathcal{R}_{c p}(\mathcal{A})$. For any $\epsilon>0$, there exists a run $\rho_{\epsilon}$ that follows $\pi$ such that last $\left.\left(\rho_{\epsilon}\right)\right|_{\text {cost }} \leq W(\pi)+\epsilon$.

For any $\epsilon>0$, we construct a strategy for Controller in $\mathcal{A}$ that achieves cost value $\left(\mathcal{R}_{\mathrm{cp}}^{\mathrm{np}}(\mathcal{A}), \ell\right)+O(\epsilon+\delta)$, so this will be equal to value $\left(\mathcal{R}_{\mathrm{cp}}^{\mathrm{np}}(\mathcal{A}), \ell\right)+$ $O(\epsilon)$ in the limit, hence the infimum is value $\left(\mathcal{R}_{\mathrm{cp}}^{\mathrm{np}}(\mathcal{A}), \ell\right)$ as required. Assume that value $\left(\mathcal{R}_{\mathrm{cp}}^{\mathrm{np}}(\mathcal{A}), \ell\right)$ is finite. Let $\pi$ denote a non-punctual path of $\mathcal{R}_{\mathrm{cp}}^{\mathrm{np}}(\mathcal{A})$ with $W(\pi)=\operatorname{value}\left(\mathcal{R}_{\mathrm{cp}}^{\mathrm{np}}(\mathcal{A}), \ell\right)$. By Lemma 10 , there exists a run $\rho_{\epsilon}$ in $\mathcal{A}$ that follows $\pi$, with last $\left.\left(\rho_{\epsilon}\right)\right|_{\text {cost }} \leq W(\pi)+\epsilon$. If all delays in $\rho_{\epsilon}$ are positive, then let $\epsilon_{0}>0$ smaller than any delay and such that all timed traces $\operatorname{Ball}_{d}\left(\operatorname{ttrace}\left(\rho_{\epsilon}\right), \epsilon_{0}\right)$ induce valid runs that follow $\pi$. Here, such an $\epsilon_{0}>0$ exists since ttrace $(\pi)$ is an open set (Proposition 2). Let $\delta \leq \epsilon_{0}$. Then, in $\mathcal{G}_{\delta}^{c}(\mathcal{A})$, if Controller suggests the delays and actions of ttrace $(\pi)$, any outcome $\rho$ is a valid run that follows $\pi$. Moreover, since each delay can be perturbed at most by $\delta$, we have last $\left.(\rho)\right|_{\text {cost }} \leq$ last $\left.\left(\rho_{\epsilon}\right)\right|_{\text {cost }}+\delta|\pi| S \leq W(\pi)+\epsilon+\delta|\pi| S$, where $S$ is the maximal slope of the cost at locations visited by $\pi$. The lemma follows.

If not all delays of $\rho_{\epsilon}$ are positive, then we choose another run $\rho_{\epsilon}^{\prime}$ with this property, such that $d\left(\operatorname{ttrace}\left(\rho_{\epsilon}\right), \operatorname{ttrace}\left(\rho_{\epsilon}^{\prime}\right)\right) \leq \epsilon$. This is possible since ttrace $(\pi)$ is open. In this case, the above proof yields a run $\rho$ with last $\left.(\rho)\right|_{\operatorname{cost}} \leq W(\pi)+$ $2 \epsilon+\delta|\pi| S$.

If $\operatorname{value}\left(\mathcal{R}_{\mathrm{cp}}^{\mathrm{np}}(\mathcal{A}), \ell\right)=\infty$, then $\ell$ is not reachable by any run, so $\lim$-value ${ }^{\text {cons }}(\mathcal{A}, \ell)=\infty$. If value $\left(\mathcal{R}_{\mathrm{cp}}^{\mathrm{np}}(\mathcal{A}), \ell\right)=-\infty$, then one can construct the above strategy for any $M<0$, considering a path $\pi$ with $W(\pi)<M$, which yields lim-value ${ }^{\text {cons }}(\mathcal{A}, \ell)=-\infty$.

Now, the polynomial-space algorithm consists in guessing a path in $\mathcal{R}_{\mathrm{cp}}^{\mathrm{np}}(\mathcal{A})$ on-the-fly, that reaches a given target location with cost less than a given bound. Such an exploration can be done in polynomial space, since the states of the corner-point abstraction can be stored, and their successors can be computed in polynomial space. See also [8].

\section{B Details of Subsection 4.2}

This section contains some modules and their properties that were omitted in the core of the paper. 
Figure 7 defines one half of the module Reduce $_{\boldsymbol{k}}(x, y, u, t)$, named PReduce $_{\boldsymbol{k}}(x, y, u, t)$. Any run from $\ell_{1}$ to $\ell_{2}$ leaves the encoded configuration unchanged, but only modifies the shifts. This automaton reduces the shift of the clocks $x, y$ (to 3 and 2 , respectively) but the shifts of $x^{\prime}, y^{\prime}$ increase by 5 . The whole module $\operatorname{Reduce}_{\boldsymbol{k}}(x, y, u, t)$ is defined by concatenating $\operatorname{PReduce}_{\boldsymbol{k}}(x, y, u, t)$ and PReduce $\boldsymbol{k}_{\boldsymbol{k}}\left(x^{\prime}, y^{\prime}, t, u\right)$, by merging the location $\ell_{2}$ of the former with the location $\ell_{1}$ of the latter. Note that $u$ and $t$ switch roles at the end of each PReduce $\boldsymbol{k}_{\boldsymbol{k}}(\cdot)$; in fact, $u, u^{\prime}$ are still needed at location $\ell_{2}$ to verify that there has been no perturbation, so they cannot both be reset. Module PReduce $\boldsymbol{k}_{\boldsymbol{k}}$ satisfies the following property, which implies Lemma 2.

Lemma 11. Let $\delta<\frac{1}{2}$. Assume PReduce $(x, y, u, t)$ is entered with valuation $\nu \in \operatorname{code}_{\boldsymbol{k}}^{\epsilon}(n, m)$ for some $\epsilon<\frac{1}{2}$. Controller has a strategy to either go to the target location with cost $-\infty$ or to reach location $\ell_{2}$ with valuation $\nu^{\prime}$ satisfying $\nu^{\prime}=$ fracp $(\nu)+\boldsymbol{k}^{\prime}$ where $\boldsymbol{k}^{\prime}$ is defined as follows: $k_{x}^{\prime}=2, k_{x^{\prime}}^{\prime}=k_{x^{\prime}}+2, k_{y}^{\prime}=$ $1, k_{y}^{\prime}=k_{y^{\prime}}+5$.

In the above lemma, the bound on $\delta$ and $\epsilon$ ensure that valuations in $\operatorname{code}_{\boldsymbol{k}}^{\epsilon}(n, m)$, when perturbed by $\delta$ differ by less than an integer from the exact valuation $\operatorname{code}_{\boldsymbol{k}}(n, m)$.

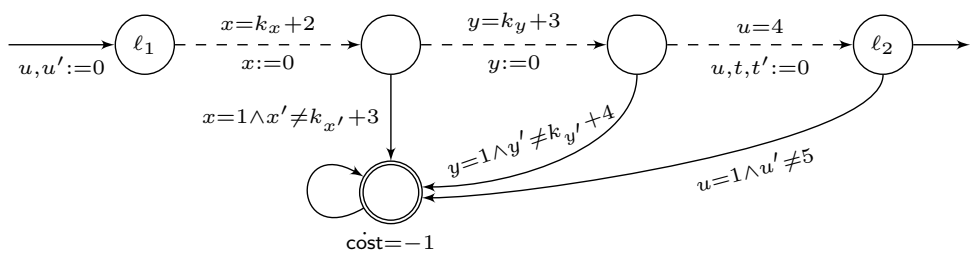

Fig. 7. Module PReduce $\boldsymbol{k}_{(}(x, y, u, t)$, reducing the shifts of $x$ and $y$.

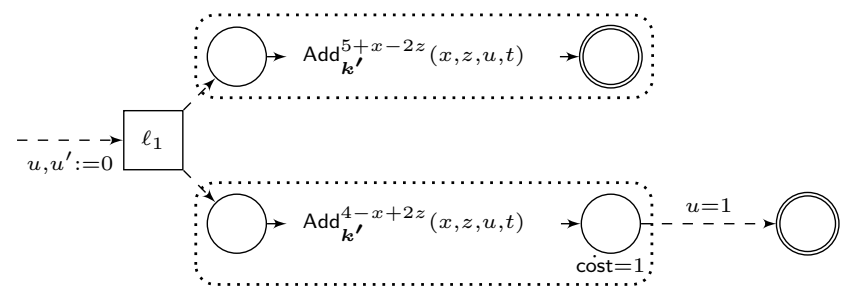

Fig. 8. Module Test ${ }^{2 z=x}(x, z, u, t)$ obtained by letting Perturbator choose between $\operatorname{Add}_{\boldsymbol{k}^{\prime}}^{5+x-2 z}(x, z, u, t)$ and $\operatorname{Add}_{\boldsymbol{k}^{\prime}}^{5+2 z-x}(x, z, u, t)$. The latter module is defined by appending one unperturbed transition with forcing the game to spend exactly one time unit at the last location of $\operatorname{Add}_{k^{\prime}}^{4+2 z-x}(x, z, u, t)$.

Proof (of Lemma 6). We let Controller go to the target location after unperturbed edges, whenever there has been a nonzero perturbation. So, to describe 
Controller's strategy, it suffices to define its delay in location $B$ since the other edges are deterministic. We let Controller delay $2-\frac{\operatorname{fracp}(\nu(x))}{2}$ in $B$. This delay can be perturbed by $\delta$. Then, the delay at location $C$ must be $1+\frac{\operatorname{fracp}((\nu(x))}{2} \pm \delta$. Upon arrival to $D$, Perturbator either chooses to go to the module Reduce $\boldsymbol{k}^{\prime}$ and the play continues at $\operatorname{Instr}_{\boldsymbol{k}^{\prime \prime}}^{j}$, or it chooses to the test module. By Lemma 5, the outcome is then at most $5+2 \delta$.

Now, if the game proceeds to $\operatorname{Instr}_{k}{ }^{j}$, then the encoding for counter $m$ has not changed thanks to the unperturbed edges. For counter $n$, we have initially $\operatorname{fracp}(x)=\frac{1}{2^{n}}+\eta$ with $\eta \in[-\epsilon, \epsilon]$. We get that upon arrival to $D$, we must have $\operatorname{fracp}(z)=\frac{1}{2^{n+1}}+\eta / 2 \pm \delta$. Hence $\nu^{\prime} \in \operatorname{code}_{\boldsymbol{k}^{\prime}}^{\delta+\epsilon / 2}(n+1, m)$, where $\nu^{\prime}$ denotes the valuation upon arrival to $D$.

Decrementation Module Aut ${ }_{k}{ }^{c_{1},-}$. We now define a module that simulates the decrementation with zero-test instruction. We explain the expected behavior of the module. The zero-test is done at the first location $A$ : the counter $c_{1}$ equals 0 if exactly one time unit after the arrival at $A$, we have $x=k_{x}+2$. Recall that the encoding is exact for counters having the value 0 . Otherwise, the run moves to either $B$ or $C^{\prime}$. In the former, Controller is to wait for $1-2 \operatorname{fracp}(x)$ time units, so that $z=1+2 \operatorname{fracp}(x)$ upon arrival to $D$. This simulates a decrementation of $c_{1}$, up to an error of $\delta$, since the edge from $B$ to $C$ can be perturbed. If the decremented value of the counter $c_{1}$ is 0 , then the new value for $z$ can be chosen as exactly 2 by Controller along the path through $C^{\prime}$.

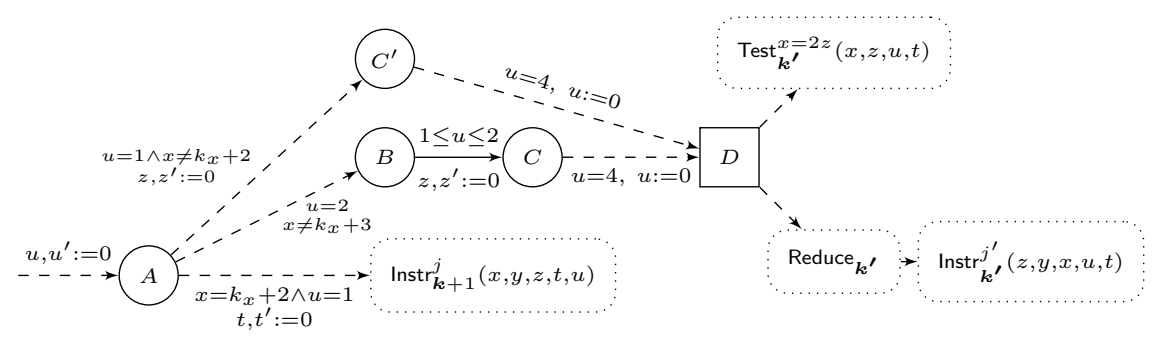

Fig. 9. Module $\operatorname{Aut}_{k}{ }^{c_{i},-}(x, y, z, u, t)$ with zero test. The module Reduce ${ }_{k^{\prime}}$ refers to Reduce $_{\boldsymbol{k}^{\prime}}(z, y, u, t)$.

Proof (of Lemma 7). If $n=0$, then $\nu(x)=k_{x}+1$. In this case, Controller can choose to move, after a delay of 1, to $\operatorname{Instr}_{k}{ }^{j}$. Controller wins by moving to a target location if Perturbator perturbs, otherwise the play continues in $\operatorname{Instr}_{k}{ }^{j}$.

If $n \geq 1$ and $\epsilon<\frac{1}{2}$, then $x \in k_{x}+\frac{1}{2^{n}} \pm \frac{1}{2}$, which means $x<k_{x}+1$. Therefore the guards of the edge leading to $\mathrm{Aut}_{k}{ }^{j}$ is never enabled. Controller can go to $B$, where it delays $1+2 \operatorname{fracp}(\nu(x))$. Due to perturbations, it will delay $1+2 \operatorname{fracp}(\nu(x)) \pm \delta$ at $C$. Thus, upon arrival to location $D$, the valuation satisfies $\operatorname{fracp}(z) \in \frac{1}{2^{n-1}} \pm 2 \epsilon \pm \delta$. By Lemma 5 , the cost increases by at most $5+2 \delta$ from here if the play goes into the test module. If $n=1$, then Controller can 
go through $C^{\prime}$ and end in $D$ with $\operatorname{fracp}(z)=2$. Here, the the test module can increase the cost by at most $5+\epsilon+2 \delta$.

Proof (of Proposition 1). Assume that $M$ halts, say after $N$ steps. Since we are interested in the limit, fix any $\epsilon>0$ and assume that $0<3^{N} \delta<\epsilon$. We let Controller play respecting the encoding: In modules Aut ${ }_{k}{ }^{c_{i},+}$, it applies the strategy of Lemma 6 to increment the counter $c_{i}$. In modules Aut ${ }_{k}{ }^{c_{i},-}$, it applies the strategy of Lemma 7 to decrement the counter $c_{i}$. Note that this strategy does not depend on $\delta$. At most $N$ modules Aut ${ }_{k}{ }^{c_{i}, \cdot}$ are visited since the target location is reached afterwards. Thus, by Lemma 12, along this play the configuration always belongs to $\operatorname{code}_{\boldsymbol{k}}^{\epsilon^{\prime}}(n, m)$ for some $\boldsymbol{k}$ with $\epsilon^{\prime} \leq \epsilon$. By these lemmas, either the play simulates the whole path of $M$ and ends in a target location, or it reaches a target sink location (with cost $=-1$ ) earlier. This can be due either to a perturbation by Perturbator along unperturbed edges, or to Perturbator's choice to go to a test module. In both cases the cost is at most $5+\epsilon+5 \delta \leq 5+2 \epsilon$, hence the limit is 5 .

Assume now that $M$ does not halt. Fix any strategy $\sigma$ of Controller. If $\sigma$ respects the encoding, i.e. always chooses $z$ as $\frac{\operatorname{fracp}(x)}{2}$ or $2 \operatorname{fracp}(x)$ in $\operatorname{Aut}_{\boldsymbol{k}_{c_{i}},+}$ or Aut $\boldsymbol{k}_{\boldsymbol{c}_{i},-}$ respectively, then the cost is $\infty$ if Perturbator never perturbs. Suppose now that $\sigma$ "cheats" during the simulation at least once. We describe a strategy for Perturbator that leads to a limit cost greater than 5. Perturbator does not perturb the run, and waits until the first instant where Controller does not respect the encoding. This happens inside Aut $\boldsymbol{k}^{c_{i},+}$ or $\mathrm{Aut}_{\boldsymbol{k}}{ }^{c_{i},-}$ along a perturbed edge since all other edges are deterministic. Assume for instance the run under strategy $\sigma$ arrives to location $D$ of Aut $_{\boldsymbol{k}_{i},+}$ with $z=1+\frac{\text { fracp }(x)}{2}+\eta$ for some $\eta$ depending on $\sigma$. Then, Perturbator chooses to go to the test module, which accepts with cost at least $5+\eta$, by Lemma 5 . Here, $\eta$ only depends on the strategy $\sigma$, so the limit $\lim -\operatorname{cost}_{\sigma}^{\text {exs }}\left(\mathcal{A}_{M}\right)$ is at least $5+\eta>5$.

The following is used in the above proof, to bound the accumulation of the error in the encoding.

Lemma 12. Consider the two functions $f: x \mapsto 2 x+1$ and $g: x \mapsto x / 2+1$. For any $n \geq 1, x>0$, and any $f_{1}, \ldots, f_{n} \in\{f, g\}, f_{1} \circ f_{2} \circ \ldots \circ f_{n}(x) \leq 3^{n} x$.

\section{Undecidability on Weighted Timed Games}

We prove Theorems 3 and 4 using a reduction similar to the one in Section 4.2. In this section, strategies and outcomes refer to the conservative perturbation game semantics.

Test Module. We define the new module $\operatorname{Add}_{k}^{\prime 1+x}(x, u, t)$ depicted in Fig. 10. We do not have any guards on the edges; Controller checks at the last step whether all delays were chosen as expected. Recall that the dotted edges are uncontrollable edges, so they are taken by Perturbator. Here, there is no upper bound on the delays that can be suggested by Perturbator, so the cost can increase arbitrarily. However, the target sink state has a negative slope on variable cost, so the cost is $-\infty$ whenever Perturbator "cheats". 


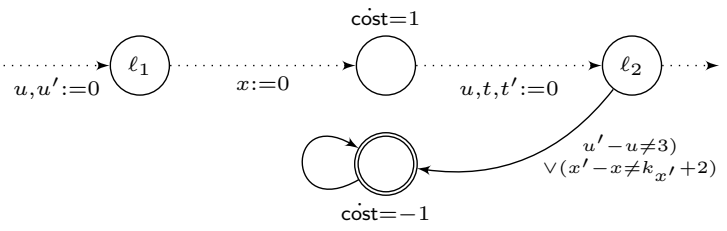

Fig. 10. Module $\operatorname{Add}_{\boldsymbol{k}}^{\prime 1+x}(x, u, t)$.

We define similarly modules $\operatorname{Add}^{\prime 2-x}(x, u, t)$. As previously, these modules can be combined to define $\operatorname{Add}^{\prime 5+x-2 z}(x, z, u, t)$. Since it is now Perturbator's duty to respect the encoding, Controller will test the chosen values using the Test $_{\boldsymbol{k}}{ }_{\boldsymbol{k}}^{x=2 z}(x, z, u, t)$ module, in Fig. 11.

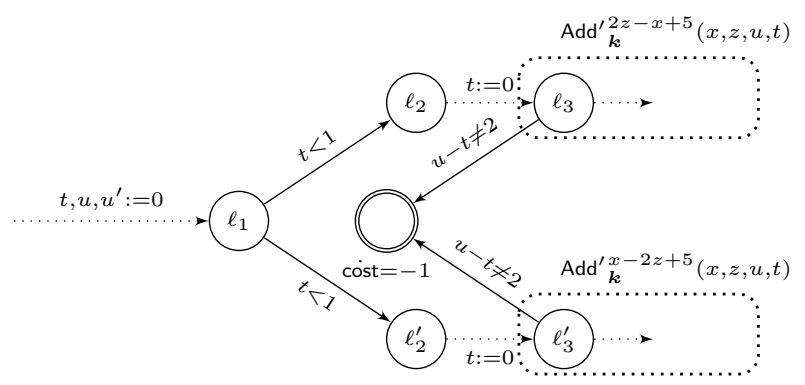

Fig. 11. Module Test ${ }_{\boldsymbol{k}}^{\prime x=2 z}(x, z, u, t)$.

Lemma 13. Assume that module Test' ${ }_{\boldsymbol{k}}^{x=2 z}(x, z, u, t)$ is entered at $\ell_{1}$ with valuation $\nu \in \operatorname{code}_{\boldsymbol{k}}^{\epsilon}$ for some $0<\epsilon<\frac{1}{2}$. Then, Controller has a strategy to reach a target location with cost at most $5-|\operatorname{fracp}(\nu(x))-2 \operatorname{fracp}(\nu(z))|$.

Incrementation Module Aut ${ }_{k}^{c_{i},+}$. Now, the module Aut $_{k}^{c_{1},+}(x, y, z, u, t)$ that increments counter $c_{1}$, given in Fig. 6 , is defined similarly to the excess-perturbation case, by exchanging the roles of the players. Along this module, if Perturbator does not get the right value for $z$ (which is $z=1+\operatorname{fracp}(x) / 2$ ), then Controller can go to the test module and reach the target location with cost $5-|2 \operatorname{fracp}(z)-\operatorname{fracp}(x)|$; it can also continue to $\operatorname{Instr}_{\boldsymbol{k}}{ }^{\prime j}$. We omit the definition of Reduce $\boldsymbol{k}^{\prime}$ but it can be adapted from Reduce $_{\boldsymbol{k}}$ without difficulty, similarly to $\operatorname{Add}_{\boldsymbol{k}}{ }_{\boldsymbol{k}}$ modules: Perturbator determines the run, and Controller checks it afterwards. The module Aut $_{k}^{\prime c_{2},+}(x, y, z, u, t)$ is defined similarly by exchanging the roles of $x$ and $y$.

We state the properties of module Aut $_{k}^{\prime c_{1},+}(x, y, z, u, t)$ more generally since we would like Controller to tolerate a bounded error in Perturbator's move:

Lemma 14. Assume that module Aut ${ }_{k}^{c_{1},+}(x, y, z, u, t)$ is entered at $A$ with valuation $v \in \operatorname{code}_{\boldsymbol{k}}^{\epsilon}(n, m)$ with $\epsilon<\frac{1}{4}$. For any $\eta>0$, Controller has a strategy 


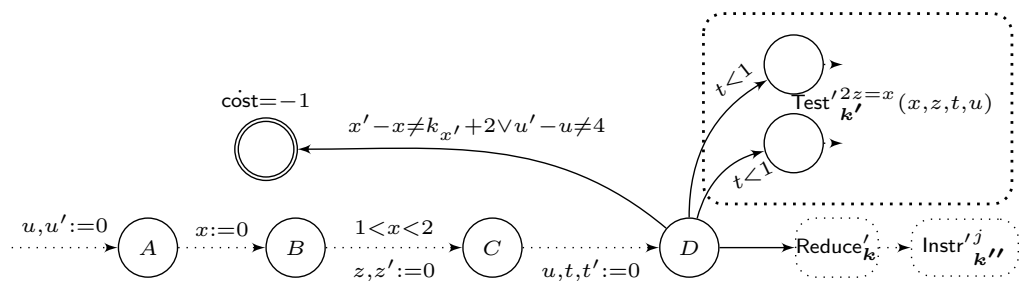

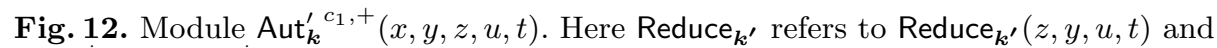
Instr $_{\boldsymbol{k}^{\prime \prime}}^{j}$ to $\operatorname{Instr}_{\boldsymbol{k}^{\prime \prime}}^{j}(z, y, x, u, t)$.

to either reach a target location with cost at most $5-\eta$, or reach $\operatorname{Instr}_{\boldsymbol{k}}{ }^{j}$ with a valuation $\nu^{\prime} \in \operatorname{code}_{\boldsymbol{k}^{\prime}}^{\epsilon / 2+\eta}(n+1, m)$.

Proof. Fix $\eta>0$. The strategy is the following. If upon arrival to $D$, the valuation $\nu^{\prime}$ satisfies $\left|2 \operatorname{fracp}\left(\nu^{\prime}(z)\right)-\operatorname{fracp}(\nu(x))\right|>\eta$, then Controller goes to the test module. Otherwise, it proceeds to $\operatorname{Instr}_{\boldsymbol{k}}^{\prime j}$ after going through the Reduce $\boldsymbol{k}_{\boldsymbol{k}}$.

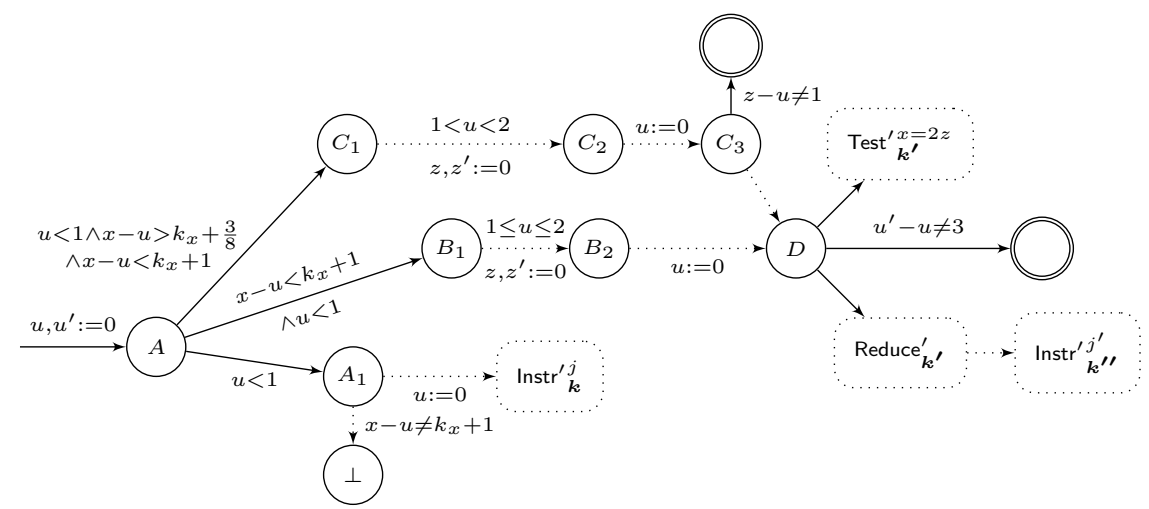

Fig. 13. Module Aut $_{\boldsymbol{k}}^{c_{1},-}(x, y, z, u, t)$ : decrementation with zero test.

Decrementation Module Aut ${ }_{k}^{c_{1},-}$. The module Aut ${ }_{k}^{c_{1},-}(x, y, z, u, t)$, given in Fig. 13, is also an adaptation of $\operatorname{Aut}_{\boldsymbol{k}}^{c_{1},-}(x, y, z, u, t)$ of the excess-perturbation case.

Lemma 15. Assume module Aut ${ }_{\boldsymbol{k}}^{c_{1},-}$ is entered with valuation $\nu \in \operatorname{code}_{\boldsymbol{k}}^{\epsilon}(n, m)$ and cost 0 , with $\epsilon<\frac{1}{4}$. Then,

- If $n=0$, then Controller has a strategy to ensure reaching either $\operatorname{Instr}_{k}{ }_{k}^{j}$ with the same configuration and cost, or an accepting location with cost $-\infty$. In this case, Controller cannot reach Instr ${ }_{\boldsymbol{k}^{\prime \prime}}^{j^{\prime}}$.

- If $n \geq 1$, then Controller cannot reach $\operatorname{Instr}_{\boldsymbol{k}}{ }_{\boldsymbol{k}}$. 
- If $n \geq 1$, for any $\eta>0$, Controller has a strategy that ensures that either Instr ${ }^{j^{\prime}}{\boldsymbol{\boldsymbol { k } ^ { \prime \prime }}}^{\prime \prime}$ is reached with valuation $\nu^{\prime} \in \operatorname{cod} e_{\boldsymbol{k}^{\prime}}^{2 \epsilon+\eta}(n-1, m)$ for some $\boldsymbol{k}^{\prime}$, or the target location is reached with cost at most $5-\eta$.

Proof. Recall that, by definition, for any $\nu \in \operatorname{code}_{\boldsymbol{k}}^{\epsilon}(0, m), \nu(x)=k_{x}$. The module $\operatorname{Instr}_{\boldsymbol{k}}^{\prime j}$ is reachable if, and only if $n=0$ since Perturbator can lead to the nonaccepting sink state $(\perp)$ whenever Controller moves to $A_{1}$ while $\nu(x) \neq k_{x}$; otherwise $\operatorname{Instr}^{\prime j}$ is reached. The guard of the other edges from $A$ are not satisfied since $x-u=k_{x}+1$.

If $n=1$, Controller can go to $C_{1}$. In fact, since $\epsilon<\frac{1}{4}$, the guard $x-u>k_{x}+\frac{3}{8}$ is satisfied. Then, the path from $C_{1}$ to $D$ forces Perturbator to ensure $z-u=1$. If Controller goes to $\operatorname{Instr}_{\boldsymbol{k}^{\prime \prime}} j^{\prime}$, this condition yields a valuation in $\operatorname{code}_{\boldsymbol{k}^{\prime \prime}}^{\epsilon}(0, m)$. If $n \neq 1$, then the guard of the edge from $A$ to $C_{1}$ is not satisfied by the assumption on $\epsilon$.

When $n \geq 1$, Controller goes to $B_{1}$, from where Perturbator is expected to lead to $D$ with some valuation $\nu^{\prime}$ satisfying $\nu^{\prime}(z)=2 \operatorname{fracp}(\nu(x)) \pm \alpha$, for some $\alpha$. Now, if $|\alpha| \geq \eta$, we let Controller go to the test module, therefore, end in an accepting location with cost at most $5-\eta$ (by Lemma 13). Otherwise, it proceeds to Instr" ${ }_{\boldsymbol{k}^{\prime \prime}}$ j. $^{\prime}$

Complete Reduction. Let $\mathcal{A}_{M}^{\prime}$ denote the weighted timed game constructed in this manner for a machine $M$. Let $\ell$ denote the target location obtained by merging all target location of the above modules. Theorems 3 and 4 follow from the following proposition.

Proposition 3. Minsky machine $M$ halts if, and only if

$$
\inf _{\sigma \in S_{C}\left(\mathcal{G}_{\delta}^{c}(\mathcal{A})\right)} \lim -\operatorname{cost}_{\sigma}^{\text {cons }}\left(\mathcal{A}_{M}^{\prime}, \ell\right)<5
$$

if, and only if for all $\delta \in\left[0, \frac{1}{3}\right]$,

$$
\inf _{\sigma \in S_{C}\left(\mathcal{G}_{\delta}^{c}(\mathcal{A})\right)} \sup _{\sigma^{\prime} \in S_{P}\left(\mathcal{G}_{\delta}^{c}(\mathcal{A})\right)} \operatorname{cost}_{\sigma, \sigma^{\prime}}^{\ell}\left(\mathcal{A}_{M}^{\prime}\right)<5,
$$

if, and only if for all $\delta \in\left[0, \frac{1}{3}\right]$,

$$
\inf _{\sigma \in S_{C}\left(\mathcal{G}_{\delta}^{e}(\mathcal{A})\right)} \sup _{\sigma^{\prime} \in S_{P}\left(\mathcal{G}_{\delta}^{e}(\mathcal{A})\right)} \operatorname{cost}_{\sigma, \sigma^{\prime}}^{\ell}\left(\mathcal{A}_{M}^{\prime}\right)<5,
$$

Proof. Assume that the machine halts, say in $N$ steps. For any $0<\epsilon<\frac{1}{4}$, we fix $0<\eta<\epsilon \times 3^{-N}$, and assume that $0 \leq \delta \leq \eta$. Controller follows the strategy given by Lemmas 14 and 15 , respectively, in modules Aut $_{k}^{c_{i},+}$ and $\mathrm{Aut}_{\boldsymbol{k}}^{\prime c_{i},-}$. Thus, Controller goes to the test module if and only if the error in the incrementation or decrementation is more than $\eta$, otherwise it continues the simulation. Then by Lemma 12, along any play, upon arrival to these modules the valuation belongs to $\operatorname{code}_{\boldsymbol{k}}^{\epsilon}(n, m)$ for some $n, m$. Thus, Controller reaches the target location with 
cost at most $5-\eta$. Note that $\delta$ has no effect here as long as it is less than $\frac{1}{2}$. In fact, the only edges that belong to Controller either yield to target locations, or the amounts of delays are irrelevant since exact timing is ensured by Perturbator in subsequent transitions.

Assume now that the machine does not halt. In this case, for any strategy of Controller, we let Perturbator respect the encoding exactly. The play is then either never reaches a target location, or it goes inside a test module and ends in a target location with cost 5 . 